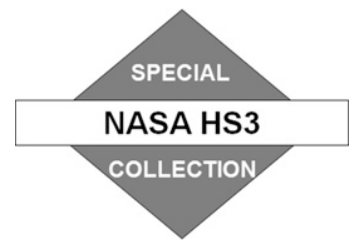

\title{
The Inner-Core Temperature Structure of Hurricane Edouard (2014): Observations and Ensemble Variability
}

\author{
ERIN B. MUNSELL \\ Laboratory for Mesoscale Atmospheric Processes, NASA Goddard Space Flight Center, Greenbelt, \\ and Universities Space Research Association, Columbia, Maryland
}

FUQING ZHANG

Department of Meteorology and Atmospheric Science, and Center for Advanced Data Assimilation and Predictability Techniques, The Pennsylvania State University, University Park, Pennsylvania

\author{
SCOTT A. BRAUN
}

Laboratory for Mesoscale Atmospheric Processes, NASA Goddard Space Flight Center, Greenbelt, Maryland

JASON A. SIPPEL

NOAA/Atlantic Oceanographic and Meteorological Laboratory/Hurricane Research Division, Miami, Florida

\author{
ANTHONY C. DiDLAKE \\ Department of Meteorology and Atmospheric Science, The Pennsylvania State University, University Park, Pennsylvania
}

(Manuscript received 11 April 2017, in final form 17 October 2017)

\begin{abstract}
The inner-core thermodynamic structure of Hurricane Edouard (2014) is explored, primarily through an examination of both high-altitude dropsondes deployed during NASA's Hurricane and Severe Storm Sentinel (HS3) and a 60-member convection-permitting ensemble initialized with an ensemble Kalman filter. The 7-day forecasts are initialized coincident with Edouard's tropical depression designation and include Edouard's significant intensification to a major hurricane. Ten-member ensemble groups are created based on timing of near-rapid intensification (RI) onset, and the associated composite inner-core temperature structures are analyzed. It is found that at Edouard's peak intensity, in both the observations and the simulations, the maximum inner-core perturbation temperature $(\sim 10-12 \mathrm{~K})$ occurs in the midlevels $(\sim 4-8 \mathrm{~km})$. In addition, in all composite groups that significantly intensify, the evolution of the area-averaged inner-core perturbation temperatures indicate that weak to moderate warming (at most $4 \mathrm{~K}$ ) begins to occur in the low to midlevels $(\sim 2-6 \mathrm{~km}) \sim 24-48 \mathrm{~h}$ prior to RI, and this warming significantly strengthens and deepens (up to $\sim 8 \mathrm{~km}$ ) $\sim 24 \mathrm{~h}$ after RI has begun. Despite broad similarities in the evolution of Edouard's warm core in these composites, variability in the height and strength of the maximum perturbation temperature and in the overall development of the inner-core temperature structure are present among the members of the composite groups (despite similar intensity time series). This result and concomitant correlation analyses suggest that the strength and height of the maximum perturbation temperature is not a significant causal factor for RI onset in this ensemble. Fluctuations in inner-core temperature structure occur either in tandem with or after significant intensity changes.
\end{abstract}

\section{Introduction}

Tropical cyclones (TCs) are frequently distinguished from extratropical cyclones by differences in their

\footnotetext{
Corresponding author: Dr. Erin B. Munsell, erin.b.munsell@ nasa.gov
}

vertical structure of temperature and wind. TC vortices are "warm core," which means that the tropospheric temperature within the inner core of the cyclone is warmer than the surrounding environment. Because the tangential wind fields of TCs are nearly balanced (Willoughby 1990), thermal wind dictates that this negative radial temperature gradient balances tangential winds 
that are maximized at low levels and decrease with height. The first observational studies that attempted to determine the radial and vertical temperature structure of TCs analyzed flight-level temperature measurements at multiple altitudes of Hurricanes Cleo (1958; La Seur and Hawkins 1963), Hilda (1964; Hawkins and Rubsam 1968), and Inez (1966; Hawkins and Imbembo 1976). Using the Jordan (1958) mean sounding as a reference profile, these studies concluded that the maximum inner-core perturbation temperature typically occurred between 250 and $300 \mathrm{hPa}$, although a secondary maximum near $600-650 \mathrm{hPa}$ was observed in Inez.

Primarily because of these initial observational studies, it became widely accepted that the height of the maximum perturbation temperature (warm core) in TCs is typically confined to the upper troposphere. However, more recent studies have suggested that this may not be the case, with Stern and Nolan (2012) arguing that the inner-core temperature structure in TCs is simply not well known. This conjecture is mainly because until recently, many of the flights into TCs were performed primarily below $6 \mathrm{~km}$, and the duration of storm sampling was typically $\sim 6 \mathrm{~h}$. Halverson et al. (2006) used dropsondes deployed by NASA's DC-8 (from 11- to 12-km height) and ER-2 (from $19 \mathrm{~km}$ ) aircraft on 10 September 2001 into Hurricane Erin and found a maximum perturbation temperature (using an environmental dropsonde as a reference profile) of $11 \mathrm{~K}$ near $500 \mathrm{hPa}$. In addition, Durden (2013) composited highaltitude dropsondes from inner-core soundings of nine different storms and found that the height of the maximum perturbation temperature existed anywhere between 750 and $250 \mathrm{hPa}$.

Recent NASA field campaigns, such as the Genesis and Rapid Intensification Processes (GRIP; Braun et al. 2013) and the Hurricane and Severe Storm Sentinel (HS3; Braun et al. 2016), have attempted to address the lack of spatial and temporal sampling through the utilization of high-altitude aircraft. Stern and Zhang (2016) used dropsondes deployed during GRIP by the DC-8 throughout the lifetime of Hurricane Earl (2010) to investigate the evolution of the inner-core temperature structure and whether or not any relationship existed between the height of the maximum perturbation temperature and the intensity evolution. Utilizing an environmental reference temperature profile (measured by dropsondes deployed by NOAA's G-IV aircraft), two distinct perturbation temperature maxima of similar magnitude were constantly observed: one in the midtroposphere $(4-6 \mathrm{~km})$ and the other in the upper troposphere $(9-12 \mathrm{~km})$. In addition, no relationship was found between the height of Earl's maximum perturbation temperature and either the current intensity or subsequent intensity changes. Komaromi and Doyle (2017) examined the composite inner-core temperature structure of six different TCs using dropsondes deployed across 16 HS3 missions and also found that neither the height nor the magnitude of the warm core correlated with intensity change.

More recent modeling studies have also suggested that the height of the warm core in TCs may be lower in the troposphere than traditionally believed. Stern and Nolan (2012) and Stern and Zhang (2013a,b) performed an extensive series of idealized experiments in which the microphysics, storm size, magnitude of vertical wind shear, and intensity all varied, and they consistently obtained TCs with maximum inner-core temperature perturbations in the midlevels $(4-8 \mathrm{~km})$. Wang and Wang (2014) obtained two distinct maxima in perturbation temperature in their simulation of Super Typhoon Megi (2010): one in the midlevels (5-6 km) and the other in the upper levels $(15-16 \mathrm{~km})$. However, the upperlevel warm core did not form until a period of rapid intensification (RI) began when the storm was already at category 2 strength. In an idealized experiment of a TC in radiative convective equilibrium performed by Ohno and Satoh (2015), the inner-core maximum perturbation temperature was found to be at $\sim 9 \mathrm{~km}$ throughout much of the intensification phase, and a secondary upper-level temperature perturbation only developed once the TC reached near-major hurricane strength. Finally, in a simulation of Hurricane Earl, Chen and Gopalakrishnan (2015) found that the maximum perturbation temperature occurred at a height of $8 \mathrm{~km}$ at peak intensity.

As discussed above, a majority of recent modeling studies have suggested the presence of a midlevel maximum perturbation temperature in the inner core of TCs. In contrast, in simulations of Hurricane Wilma (2005) performed by Chen et al. (2011) and Chen and Zhang (2013), a single maximum perturbation temperature was found at $14 \mathrm{~km}$. It was also argued that the formation of this temperature perturbation at this height helped trigger Wilma's significant period of RI. This hypothesis will be explored in the case of Hurricane Edouard's (2014) near-RI ${ }^{1}$ event in this study through the use of high-altitude dropsondes, additional HS3 and satellite observations, and a convection-permitting 60-member ensemble simulation.

\footnotetext{
${ }^{1}$ Although Edouard did not officially undergo RI (according to the NHC criteria), the period of intensification was significant (a "near-RI event"). Therefore, RI timing is examined in this ensemble as it is traditionally defined because it is more straightforward to do so.
} 
Hurricane Edouard was a named tropical cyclone from 11 to 19 September 2014 that remained over the open Atlantic Ocean throughout its lifetime (Stewart 2014). The tropical wave that eventually became Edouard exited the African coast on 6 September. As the broad area of low pressure tracked westward, convection increased near the center of the surface low, causing the wave to be designated as a tropical depression at 1200 UTC 11 September. Steady intensification followed, and Edouard became a tropical storm early on 12 September and a hurricane early on 14 September. Over the next $24 \mathrm{~h}$, a period of significant intensification occurred $\left(12.9 \mathrm{~m} \mathrm{~s}^{-1}\right.$ or $\left.25 \mathrm{kt}\right)$, and by 1200 UTC 16 September, Edouard reached its peak intensity with winds of $54.0 \mathrm{~m} \mathrm{~s}^{-1}(105 \mathrm{kt})$. Edouard began to weaken almost immediately thereafter as an eyewall replacement cycle (ERC) occurred, and as Edouard turned northward and northeastward, it accelerated ahead of an approaching midlatitude trough. On 18 September, Edouard turned eastward and rapidly weakened to a tropical storm as it became embedded in strong vertical wind shear associated with the midlatitude westerlies. It was subsequently reclassified as a strong posttropical cyclone early on 19 September.

In addition to undergoing a period of significant intensification, Edouard was also notable for the numerous research missions conducted, at times, simultaneously throughout its lifetime (Stewart 2014). The NOAA WP3D Hurricane Hunters conducted eight missions between 11 and 19 September, while NASA's Global Hawk performed four missions into and around Edouard throughout its lifetime, sampling the TC for up to 18 consecutive hours during each mission, as part of the 2014 campaign of HS3. In addition, the Global Hawk dropsondes were released from altitudes greater than $18 \mathrm{~km}$, which yielded some of the first high-resolution samples of inner-core TC temperature structure throughout the troposphere and lower stratosphere.

The overall goal of this study is to investigate the evolution of the inner-core temperature structure of Edouard prior to and throughout its period of significant intensification by using both the unusual variety of observations and a 60-member convection-permitting ensemble simulation generated by the Pennsylvania State University (PSU) real-time Atlantic hurricane analysis and forecast system. In particular, the ensemble simulation provides an opportunity to not only thoroughly examine the evolution of the modeled inner-core temperature structure, but also to examine the variability of the height and strength of the maximum temperature perturbation for groups of members that have similar intensity evolutions yet a variety of RI-onset times throughout the simulation.
Section 2 provides a description of the PSU real-time hurricane forecast and analysis setup and the available observations of Edouard's inner-core temperature structure. Section 3 presents an evaluation of Edouard's observed and simulated inner-core temperature structure, as well as correlation analyses that examine the ensemble variability of Edouard's warm core throughout the period of significant intensification. Finally, section 4 summarizes the main conclusions of this study.

\section{Methodology and data}

\section{a. PSU WRF-EnKF real-time Atlantic hurricane analysis and forecast system}

The 60-member ensemble simulation utilized in this study was originally a 126 -h forecast initialized at 1200 UTC 11 September by the PSU real-time Atlantic hurricane analysis and forecast system (Zhang et al. 2009, 2011; Zhang and Weng 2015; Weng and Zhang 2016). For the 2014 configuration of this system, version 3.5.1 of the Advanced Research version of the Weather Research and Forecasting Model (WRF-ARW; Skamarock et al. 2008 ) is coupled with an ensemble Kalman filter (EnKF) algorithm for data assimilation. Observations that are assimilated when available include Global Telecommunication System (GTS) conventional and reconnaissance data, superobservations generated from the airborne tail Doppler radar (TDR) on the NOAA P-3 aircraft (Weng and Zhang 2012), satellite-derived winds (Weng and Zhang 2016), and dropsondes deployed from the NOAA-National Center for Atmospheric Research (NCAR) Advanced Vertical Atmospheric Profiling System (AVAPS) collected during HS3 flights (Braun et al. 2016). Three two-way nested domains are utilized with horizontal grid spacings of 27,9 , and $3 \mathrm{~km}$, and all domains have 43 vertical levels and a model top at $10 \mathrm{hPa}$. The outermost domain is fixed, while the inner domains follow the vortex of the TC of interest. All physics configurations in WRF are the same as in Munsell et al. (2017).

The PSU WRF-EnKF system was first initialized for the invest area that eventually became Edouard at 0000 UTC 4 September, utilizing Global Forecast System (GFS) analyses. The first data assimilation cycle was performed on all three domains $12 \mathrm{~h}$ into integration, and continuous cycling occurred at 3-h intervals thereafter. The initial and lateral boundary conditions for the ensemble were generated by adding perturbations derived from the background error covariance of the WRF variational data assimilation system (Barker et al. 2004). In addition, in order to examine Edouard's inner-core temperature structure throughout its period of strong intensity, 40 of the 60 ensemble members (those that 

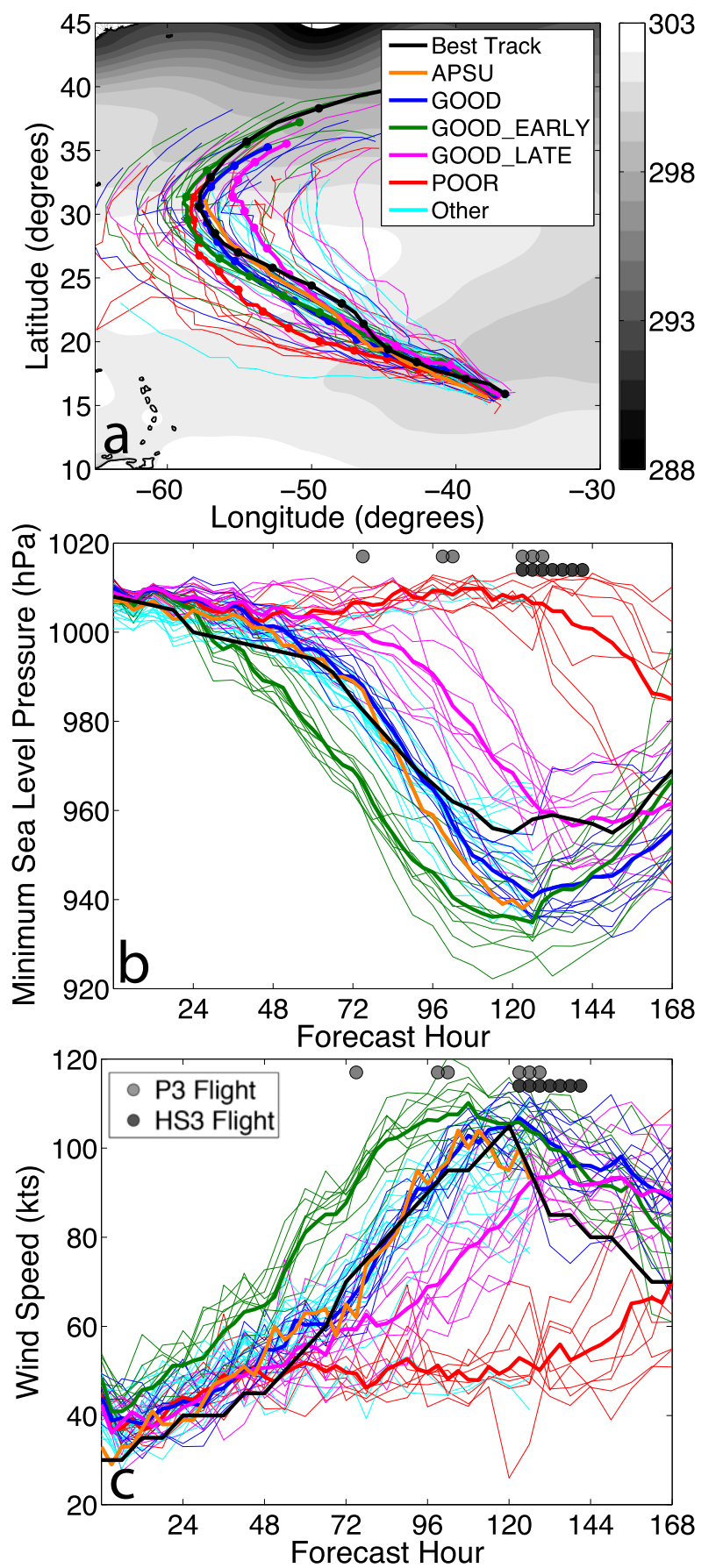

FIG. 1. A comparison of the NHC best track with deterministic and ensemble forecasts of (a) track, (b) minimum SLP (hPa), and (c) maximum $10-\mathrm{m}$ wind speed ( $\mathrm{kt}, 1 \mathrm{kt}=0.5144 \mathrm{~m} \mathrm{~s}^{-1}$ ) for the 1200 UTC 11 Sep 2014 initialization of Hurricane Edouard from the PSU WRF-EnKF system. Members are placed in composite groups of 10 according to their RI-onset time (GOOD: blue; GOOD_EARLY: green; GOOD_LATE: magenta; and POOR: red) and have been extended to 7-day forecasts (the operational real-time system only produces 126 -h forecasts). The composite means [thick; positions marked every $12 \mathrm{~h}$ in (a)], the NHC best track [black; positions marked every $12 \mathrm{~h}$ in (a)], and the 5-day comprised the composite groups in Munsell et al. 2017; detailed below) were extended an additional $42 \mathrm{~h}$ through 1200 UTC 18 September, resulting in a 168-h forecast initialized at 1200 UTC 11 September.

\section{b. Observations of Hurricane Edouard's inner-core temperature structure}

During the 2014 campaign of HS3, four flights utilizing NASA's Global Hawk were performed throughout the lifetime of Hurricane Edouard. These flights spanned Edouard's evolution from a newly formed tropical storm (11-12 September), the significant period of intensification to a strong category 2 TC (14-15 September), Edouard's maintenance near peak intensity (16-17 September), and Edouard's rapid weakening as it began to transition to an extratropical cyclone (18-19 September; Braun et al. 2016). The first two Edouard flights occurred during the original 5-day simulation window (15-27 and 72-93 h), while the third flight was performed within the 42-h extension of part of the ensemble forecast (123-141h). Observations collected during the third HS3 flight, including 87 AVAPS dropsondes (Hock et al. 2017) deployed from $\sim 18 \mathrm{~km}$ and data from the University of Wisconsin's Scanning High-Resolution Interferometer Sounder (S-HIS; Revercomb and Taylor 2017), contain information about the inner-core temperature structure of Edouard. The dropsondes have been quality controlled and postprocessed at the NCAR Earth Observing Laboratory (EOL) using NCAR's Atmospheric Sounding Processing Environment (Aspen) software (Hock et al. 2017). None of the HS3 observations were assimilated in the ensemble forecast analyzed in this study, as they were not available at the time of initialization.

Eight flights from two NOAA P-3s and a G-IV aircraft were also performed throughout Edouard's lifetime between 12 and 17 September as part of the NOAA Intensity Forecasting Experiment (IFEX; Rogers et al. 2013). This study utilizes data collected by the TDR to analyze Edouard's wind field and overall structure; dropsondes deployed by the P-3 and G-IV are not utilized to examine the inner-core temperature structure because of the P-3's significantly lower deployment altitude and the G-IV's focus on sampling the TC's environment.

APSU deterministic forecast (orange) are also plotted. The remaining ensemble members not classified in composite groups (other: cyan) remain as 5-day forecasts. Sea surface temperatures (constant throughout simulation) are contoured (filled every $1 \mathrm{~K}$ starting at $288 \mathrm{~K}$ ) in (a). The times that the NOAA P-3 (gray markers) and the 16-17 September flight of NASA's Global Hawk (dark gray markers) sampled Edouard are shown at the top of (b) and (c). 


\section{Results and discussion}

\section{a. PSU WRF-EnKF ensemble track and intensity evolution}

As the primary goal of this study is to examine the evolution of the inner-core temperature structure of Edouard throughout the period of significant intensification, the 126-h forecast chosen for analysis encompasses the TC's designation as a tropical depression through peak intensity (1200 UTC 11 September-1800 UTC 16 September). This ensemble is identical to that investigated extensively in Munsell et al. (2017), which examined the predictability and dynamics associated with the variability in RI-onset times within the ensemble. This ensemble was also used to study various other aspects of the dynamics and predictability of Edouard (Tang and Zhang 2016; Tang et al. 2017; Melhauser et al. 2017; Fang et al. 2017). Figure 1a shows the National Hurricane Center's (NHC) best track for Hurricane Edouard, as well as the deterministic APSU and ensemble members for the PSU WRF-EnKF forecast, while Figs. $1 \mathrm{~b}$ and $1 \mathrm{c}$ present the corresponding evolution of the minimum sea level pressure (SLP; in $\mathrm{hPa}$ ) and maximum 10-m wind speed (in kt). Overall, the deterministic track and intensity forecast closely follows that of the best track, and a substantial number of members $(\sim 25)$ predict an RI-onset time and rate of intensification comparable to the best track. A majority of the remaining members intensify at a similar rate as the best track; however, variability of up to $48-60 \mathrm{~h}$ in the timing of RI onset is present, with some members not intensifying at all in the 126-h forecast.

As in past ensemble sensitivity studies (e.g., Munsell et al. 2013, 2015, 2017; Munsell and Zhang 2014; RiosBerrios et al. 2016), 10-member composite groups are created according to their timing of intensification to examine the variability of the development of the innercore temperature structure. These composite groups are identical to those in Munsell et al. (2017): GOOD contains members whose RI-onset times are approximately those of the best track (1200 UTC 14 September, or 72h), GOOD_EARLY (GOOD_LATE) members undergo RI $24 \mathrm{~h}$ prior to (after) best track RI, and POOR members do not intensify substantially in the 126-h simulation window. To encompass the entirety of Edouard's peak intensity and the HS3 flight on 16-17 September (as indicated in Figs. 1 b and 1c), the 40 members that comprise these composite groups have been extended to 1200 UTC 18 September, and the resulting 168-h forecasts of track, minimum SLP, and maximum 10-m wind speed are plotted in Fig. 1. Toward the end of this new simulation window, the members of the developing composites have begun to weaken (although not as significantly as in the best track) as Edouard turns toward the northeast and into less favorable environmental conditions. However, the slower and more westward positions of the POOR members lead to some intensification after $144 \mathrm{~h}$. Much of the analysis in this study of the ensemble variability of the inner-core temperature structure evolution utilizes these composite groups, and the forecasts of the remaining 20 ensemble members ("other" in Fig. 1) were not extended. Though the evolution of the majority (15) of the "other" members resembles that of the GOOD members, the cumulative root-mean-square intensity errors are larger than in the GOOD members. The remaining five "other" members do not significantly intensify, as in POOR.

\section{b. Comparison of PSU WRF-EnKF wind field to observations}

Before analyzing the observed and modeled innercore temperature structure of Edouard in greater detail, it is useful to compare the observed horizontal and tangential wind fields to the ensemble because the structure of the tangential winds is closely related to the inner-core temperature structure through thermal wind balance. Figures 2 and 3 show storm-centered horizontal cross sections of composite $2-\mathrm{km}$ wind speed and azimuthally averaged vertical cross sections of tangential wind collected by the TDRs on the two NOAA P-3 aircrafts on 14, 15, and 16 September (Figs. 2a-c, 3a-c; flight times indicated in Figs. 1b,c) and the corresponding GOOD (Figs. 2d-f, 3d-f) and GOOD_LATE (Figs. 2g-i, 3g-i) composites from the WRF-EnKF forecast. For the TDR data, NOAA's Hurricane Research Division (HRD) performs a three-dimensional analysis of the Cartesian horizontal and vertical velocities by using the automated technique of Gamache et al. (2004). These 5-km analyses have been composited across the various legs of each $\sim 3$-h flight pattern. The observational composites in Fig. 2 are somewhat comparable to those in Rogers et al. (2016), as they utilize the same P-3 data; however, Rogers et al. (2016) uses a finer grid spacing of $2 \mathrm{~km}$, and their composites are storm relative, while the Fig. 2 composites are ground relative. The $2-\mathrm{km}$ winds as measured by the dropsondes deployed during the third HS3 flight (16-17 September) are also indicated in Fig. 2c.

The 14 September P-3 flight occurred near the beginning of Edouard's intensification from a tropical storm to a strong category 2 hurricane. The P-3 data (Fig. 2a) show that Edouard was somewhat asymmetric at this time, with the maximum 2-km winds of $\sim 40 \mathrm{~m} \mathrm{~s}^{-1}$ located to the north of the surface center. The surface 

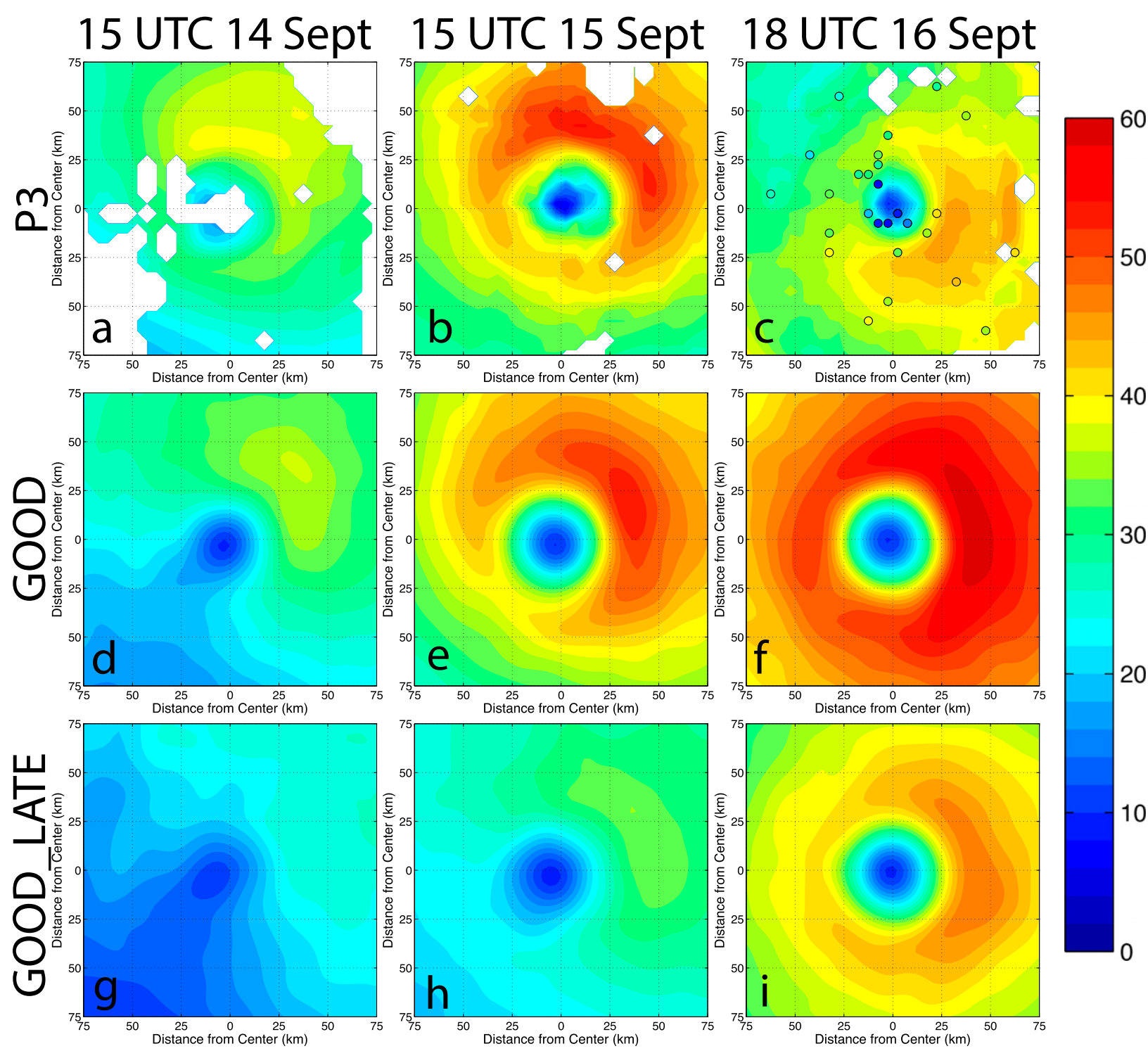

FIG. 2. Storm-centered horizontal cross sections of composite 2-km wind speed (ground relative; contours filled every $2 \mathrm{~m} \mathrm{~s}^{-1}$ ) for NOAA P-3 flights in (top) Edouard, (middle) GOOD, and (bottom) GOOD_LATE at approximately (a),(d),(g) 1500 UTC 14 Sep 2014 (75 h); (b),(e),(h) 1500 UTC 15 Sep 2014 (99 h); and (c),(f),(i) 1800 UTC 16 Sep 2014 (126 h). The 2-km wind speed as measured by the AVAPS dropsondes deployed between 1500 UTC 16 Sep and 0900 UTC 17 Sep 2014 (123-141 h) during the HS3 Global Hawk flight are indicated in (c).

radius of maximum winds (RMW) was $\sim 25 \mathrm{~km}$, while $30 \mathrm{~m} \mathrm{~s}^{-1}$ winds extended upward through a height of $\sim 8 \mathrm{~km}$ in this region (Fig. 3a). The GOOD composite at this time (Fig. 2d) simulates most of the same characteristics, though the simulated vortex is slightly more asymmetric with the maximum $2-\mathrm{km}$ winds $\left(\sim 36 \mathrm{~m} \mathrm{~s}^{-1}\right)$ located northeast of the surface center. In addition, the GOOD composite vortex has a larger RMW of $\sim 40 \mathrm{~km}$, and the vortex is slightly weaker and shallower, with $30 \mathrm{~m} \mathrm{~s}^{-1}$ wind up to only $\sim 5 \mathrm{~km}$ (Fig. 3d). It is evident in the composites from 15 September that the period of intensification was well underway, as both the P-3 data (Figs. 2b, 3b) and the GOOD composite (Figs. 2e, 3e) have maximum $2-\mathrm{km}$ winds of $\sim 55 \mathrm{~m} \mathrm{~s}^{-1}$, near-surface winds of $\sim 48 \mathrm{~m} \mathrm{~s}^{-1}$, an expanded RMW $(\sim 40 \mathrm{~km})$ that noticeably slopes outward with height, and a deep vertical extent of $30 \mathrm{~m} \mathrm{~s}^{-1}$ winds $(\sim 10-11 \mathrm{~km})$.

Despite general agreement between the P-3 data and the GOOD composites on 14 and 15 September, the composites are markedly different from the radar analyses on 16 September. The P-3 data and the dropsondes deployed during the 16-17 September HS3 flight (Fig. 2c) 

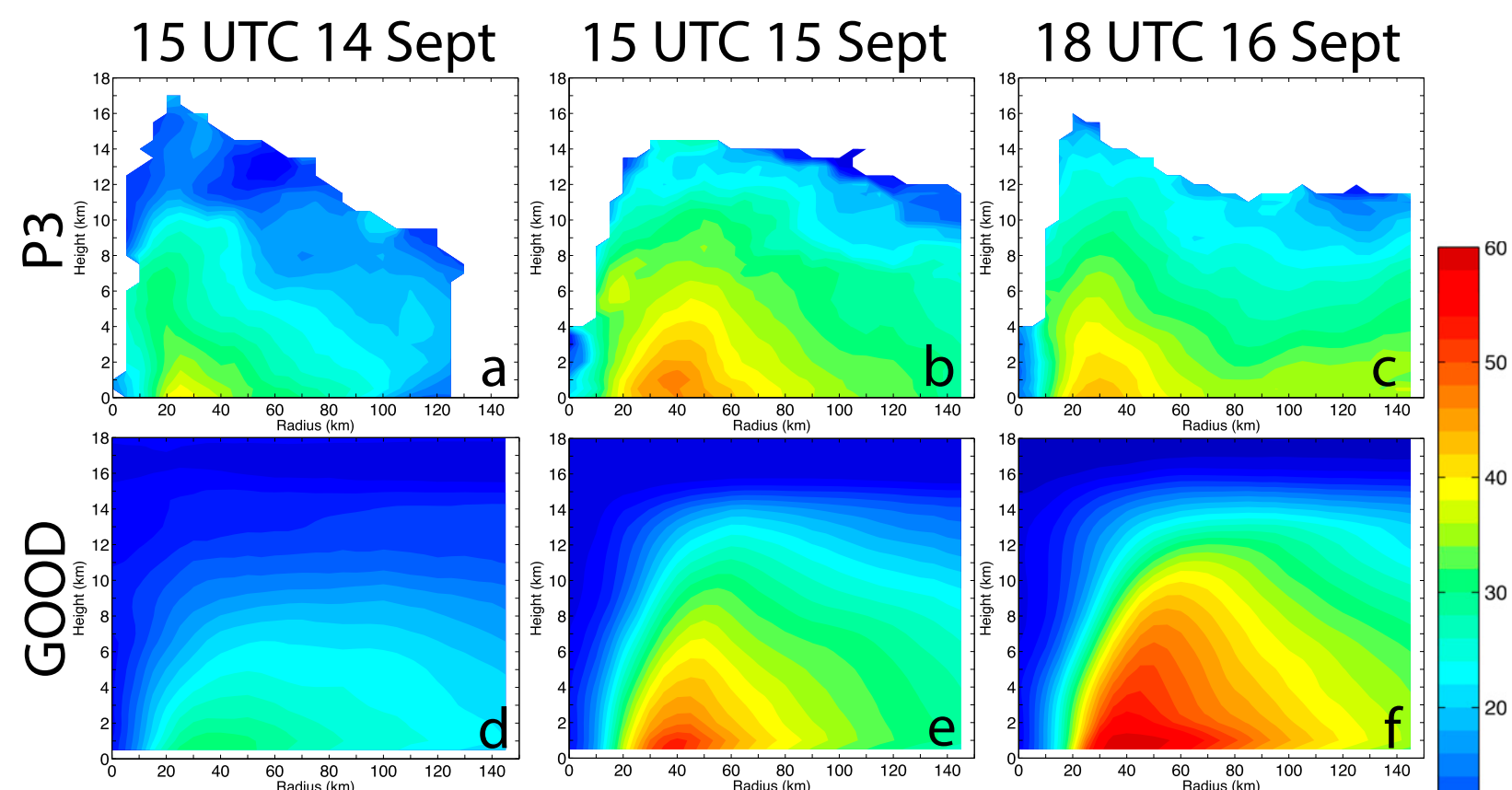

40
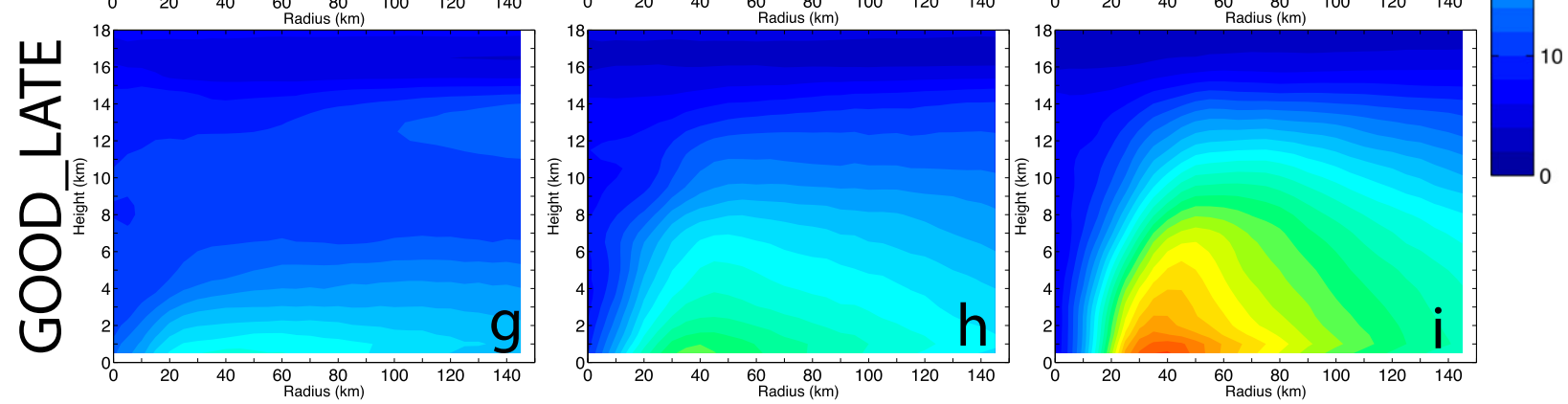

FIG. 3. As in Fig. 2, but for azimuthally averaged vertical cross sections of composite tangential winds (contours filled every $2 \mathrm{~m} \mathrm{~s}^{-1}$ ).

indicate that the vortex at $2 \mathrm{~km}$ weakened to $\sim 45 \mathrm{~m} \mathrm{~s}^{-1}$, with the strongest winds located to the southeast of the surface center. A secondary wind maximum is also apparent in the observations $\sim 50 \mathrm{~km}$ east of the center, as an ERC was occurring throughout these flights. However, the GOOD composite (Fig. 2f) shows a stronger $\left(\sim 60 \mathrm{~m} \mathrm{~s}^{-1}\right)$ and more symmetric vortex, with no evidence of a secondary wind maximum. The vertical cross section of P-3 tangential wind data (Fig. 3c) indicates that Edouard's near-surface winds $(\sim 0.5 \mathrm{~km})$ decreased somewhat to $\sim 44 \mathrm{~m} \mathrm{~s}^{-1}$, and the RMW contracted to $30 \mathrm{~km}$ and became more upright with height. Conversely, the near-surface winds of the GOOD composite vortex are significantly stronger (upward of $60 \mathrm{~m} \mathrm{~s}^{-1}$ ), the RMW remains at $40 \mathrm{~km}$, and the outward slope of the RMW has increased (Fig. 3f).

Though there is considerable disagreement between the observed and simulated composites on 16-17 September, the simulation results can still provide useful insights at this time. The disagreement results from the failure of some GOOD members to capture an ERC and also a tendency of GOOD to decay at a slower rate than observed. The GOOD_LATE composites at this time are in better agreement with the observed composites, as the minimum SLP (Fig. 1b), horizontal 2-km winds (Fig. 2i), 0.5-km tangential winds, and the RMW (Fig. 3i) are comparable. However, because of their later RI onsets, the GOOD_LATE members reach their peak intensities just prior to this time. Despite the lack of any secondary wind maxima in the composites, a closer examination of the evolution of the $1-\mathrm{km}$ tangential winds and vertical velocities reveals that a majority (14 out of 20) of the GOOD and GOOD_LATE members show evidence of an ERC (not shown). Therefore, although neither the GOOD nor GOOD_LATE members simulate the exact structural evolution of Edouard, both composite groups are able to accurately capture RI, and many members replicate the ERC in the decay phase. This allows for reasonable comparisons to the observed inner-core temperature structure. 

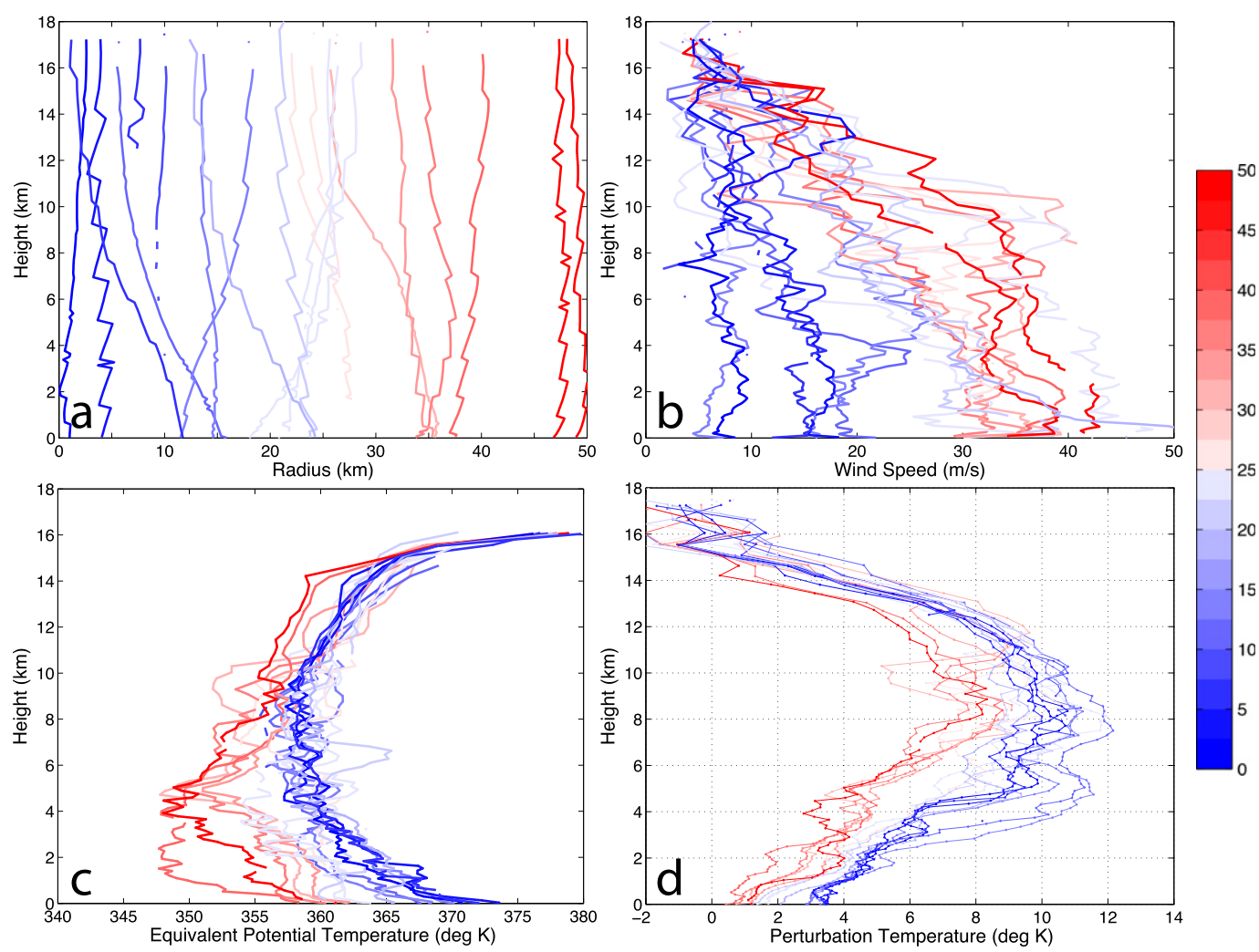

FIG. 4. Vertical profiles from the inner-core (within $50 \mathrm{~km}$ of the surface center) AVAPS dropsondes of the (a) distance from Edouard's surface center $(\mathrm{km}),(\mathrm{b})$ winds $\left(\mathrm{m} \mathrm{s}^{-1}\right)$, (c) equivalent potential temperature $(\mathrm{K})$, and (d) perturbation temperature $(\mathrm{K})$, with respect to the mean environmental reference profile calculated from the temperatures measured by the dropsondes deployed between 300 and $700 \mathrm{~km}$ from Edouard's surface center during the 16-17 September HS3 Global Hawk flight. All profiles are colored (every $5 \mathrm{~km}$ from 0 to $50 \mathrm{~km}$ ) according to the mean distance from Edouard's surface center that the dropsonde traveled.

\section{c. Analysis of the observed warm core}

The inner-core temperature structure of Edouard was only sufficiently sampled for further analysis throughout the 16-17 September flight, when Edouard was a strong category 2 storm. During this period, 87 dropsondes were deployed, with 21 of them passing within $50 \mathrm{~km}$ of the surface center $^{2}$ at some point during their descent. The positions of these inner-core dropsondes, color-coded by distance from Edouard's surface center, are shown in Fig. 4a. It should be noted that only six of these dropsondes are confined to within $20 \mathrm{~km}$ of Edouard's surface center throughout descent, which can lead to an underestimation of the magnitude of the inner-core perturbation temperature.

\footnotetext{
${ }^{2}$ The estimated storm centers are obtained from S-HIS data collected during the nine eye overpasses that the Global Hawk executed during the 16-17 September flight. These center fixes were subsequently interpolated to 2-min intervals, and the appropriate center was chosen based on the time that the dropsonde was deployed.
}

The vertical profiles of wind speed as measured by the 16-17 September inner-core dropsondes are shown in Fig. 4b. About one-third of the inner-core dropsondes have wind speeds less than $20 \mathrm{~m} \mathrm{~s}^{-1}$ throughout their vertical profile, indicating that these dropsondes likely remained within the eye of Edouard for the majority of their descent. Vertical profiles of equivalent potential temperature $\left(\theta_{e}\right.$ in $\mathrm{K}$; Fig. 4c) confirm that these inner-core dropsondes primarily remained within Edouard's eye, as higher values of $\theta_{e}$ are present throughout the profiles, peaking at $370-375 \mathrm{~K}$ near the surface. The remainder of the innercore dropsondes measured wind speeds in excess of $30-45 \mathrm{~m} \mathrm{~s}^{-1}$, particularly at low levels, and therefore likely sampled at least part of Edouard's eyewall. These dropsondes also have cooler $\theta_{e}$ profiles throughout most of the troposphere, again suggesting that they remained mostly in the eyewall throughout descent.

To calculate vertical profiles of perturbation temperature, a reference profile must first be selected. Stern and Nolan (2012) extensively discussed the various choices of reference profile; most observational and 


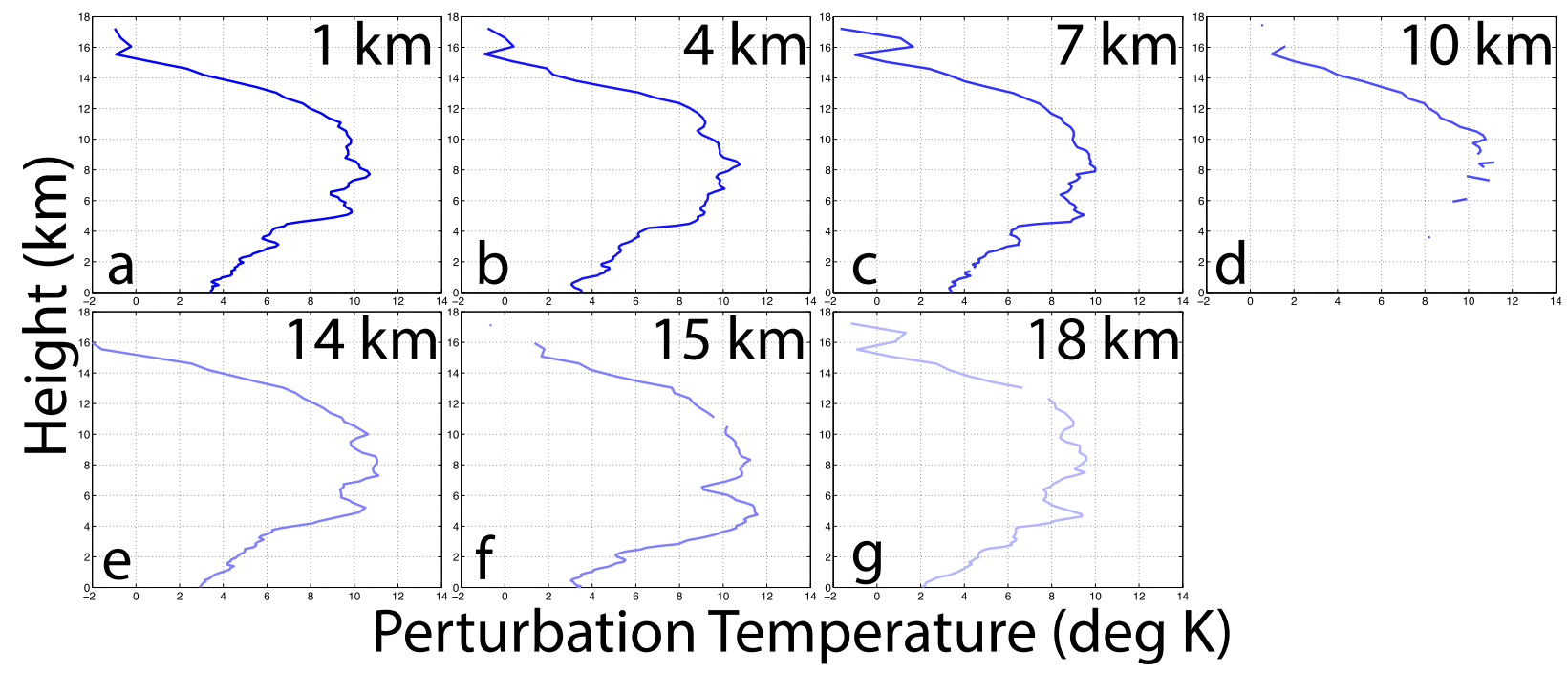

FIG. 5. As in Fig. 4d, but for only the dropsondes deployed within $20 \mathrm{~km}$ of Edouard's surface center. The seven dropsondes fell within (a) 1, (b) 4, (c), 7, (d), 10, (e) 14, (f), 15, and (g) $18 \mathrm{~km}$ throughout the 16-17 September HS3 Global Hawk flight.

modeling studies either use a mean climatological sounding, such as the Jordan (1958) or the Dunion (2011) moist tropical sounding, or a near-storm environmental profile calculated using available observational or numerical data within a specified range of distance from the surface center of the TC of interest. Durden (2013) explored the impacts of using a mean climatological (Dunion) versus a near-storm environmental reference profile and found that the resulting perturbation temperature structures were at higher altitudes and had larger magnitudes in perturbation temperature when a climatological sounding, such as Dunion (2011), was used. The Hurricane Earl perturbation temperatures calculated by Stern and Zhang (2016), in which comparisons were also made for multiple reference profiles (the Dunion sounding vs environmental profiles), were consistent with the Durden (2013) results. Therefore, this study utilizes an environmental profile as in Stern and Zhang (2016), in which the reference profile is calculated from either observations or numerical data between 300 and $700 \mathrm{~km}$ from Edouard's surface center.

Given this near-storm environmental reference profile, the resulting perturbation temperatures as measured by the inner-core dropsondes deployed throughout the 16-17 September HS3 flight are shown in Fig. 4d. From these profiles (again color-coded by the distance from Edouard's surface center), it is clear that the perturbation temperature magnitudes noticeably increase inward. In addition, there appear to be two distinct shapes of perturbation temperature profiles that also have a dependence on distance. Most of the dropsondes that were deployed closer to Edouard's surface center (within
$20 \mathrm{~km}$ of the surface center; Fig. 5) have two distinct perturbation temperature maxima: one between 4 and $6 \mathrm{~km}$ and the other between 7 and $9 \mathrm{~km}$ (Figs. 5a,c,f). Both of these near-center perturbation temperature maxima are of similar strength, $\sim 10-12 \mathrm{~K}$. A few of these dropsondes also have a third maximum of similar strength near $\sim 10 \mathrm{~km}$ (Figs. $5 \mathrm{e}, \mathrm{g}$ ). However, the majority of the dropsondes closer to Edouard's RMW ( $\sim 30 \mathrm{~km})$ have only one maximum in perturbation temperature, predominantly between heights of 7 and $9 \mathrm{~km}$. Furthermore, this single perturbation temperature maximum $(\sim 7-9 \mathrm{~K})$ is weaker than the perturbation temperature maxima that are closer to the surface center, consistent with Zawislak et al. (2016). Regardless of distance from the TC surface center, nearly all inner-core dropsondes measure decreasing perturbation temperatures above $10 \mathrm{~km}$, and there is no evidence of upper-tropospheric maxima in perturbation temperature through heights of $18 \mathrm{~km}$.

In addition to the dropsondes released on 16-17 September, additional observations of Edouard's innercore temperature structure were obtained from the airborne S-HIS and the spaceborne Advanced Microwave Sounding Unit-A (AMSU-A). Figure 6 shows a radius-height cross section of the azimuthally averaged composite inner-core perturbation temperature for Edouard for these observational sources and the GOOD_LATE members from the WRF-EnKF ensemble. All composites in Fig. 6 are calculated using the GOOD_LATE environmental reference profile averaged over a 300-700-km annulus centered on the surface center, with the exception of the AMSU-A data (Fig. 6d). The dropsonde perturbation temperatures discussed above 



FIG. 6. Radius-height cross section of azimuthal-mean perturbation temperature (K; contours filled every $0.5 \mathrm{~K}$ ) for the (a) GOOD_LATE composite at 0000 UTC 17 Sep 2014, (b) inner-core AVAPS dropsondes deployed during the 16-17 Sep 2014 HS3 Global Hawk flight, (c) S-HIS data from the same HS3 flight, and (d) CIMSSprocessed AMSU-A data from 2025 UTC 16 Sep 2014. The azimuthal-mean temperature between 300 and $700 \mathrm{~km}$ from the surface center of the GOOD_LATE composite is used as a reference profile in (a)-(c), while (d) utilizes temperature retrievals averaged at various points $\sim 500 \mathrm{~km}$ from the TC surface center.

(Fig. 4) are replotted using the modeled reference profile in Fig. 6b; similar conclusions can be drawn as from the individual vertical profiles of perturbation temperature (Fig. 4d). Two perturbation temperature maxima of $\sim 10 \mathrm{~K}$ are present in the near-center region (between 10 and $20 \mathrm{~km}$ ), while the third perturbation temperature maximum at a height of $\sim 10 \mathrm{~km}$ that was observed in some of the near-center dropsondes also is seen. At and just outside the eyewall $(\sim 40-60 \mathrm{~km}$ from the surface center), a single, weaker maximum in perturbation temperature is evident.

The azimuthal-mean perturbation temperature composite for the S-HIS data is shown in Fig. 6c. It should be noted that the capability of the S-HIS to sample the warm core is limited because this instrument cannot "see" through clouds. Within the eye region, however, where the cloud cover is reduced, a region of relatively strong $(\sim 10-11 \mathrm{~K})$ perturbation temperatures is sampled between heights of 7 and $10 \mathrm{~km}$ within radii of up to $25 \mathrm{~km}$. Therefore, the overall structure of the inner-core temperature appears to be similar between the S-HIS and dropsonde data for the 16-17 September HS3 flight, with the S-HIS analysis slightly cooler than the perturbation temperature maxima measured by the dropsondes.

In addition to direct measurements of Edouard's innercore temperature structure, remote sensing instruments on satellites also have some skill in resolving the warm core of TCs (Knaff et al. 2004); however, the lack of resolution in both the horizontal $(\sim 50 \mathrm{~km})$ and vertical (six usable channels) limits them (Stern and Nolan 2009). Figure $6 \mathrm{~d}$ shows the azimuthal-mean perturbation temperature composite of Edouard's inner core as measured by the AMSU-A multichannel microwave temperature sounder at 2025 UTC 16 September. The satellite data have been processed by the University of Wisconsin's Cooperative Institute for Meteorological Satellite Studies (UW-CIMSS); the environmental reference profile used to calculate perturbation temperatures is created from temperature retrievals at various points around the TC that are $\sim 500 \mathrm{~km}$ from the surface center. The composite perturbation temperature structure reveals two distinct maxima in perturbation temperature: one slightly higher in the atmosphere $(\sim 9-11 \mathrm{~km})$ than in either the 
dropsonde or S-HIS data, and one significantly lower in the atmosphere (near the surface). In addition, the magnitudes of these maxima are much weaker $(\sim 4 \mathrm{~K}$ for the upper maximum and $\sim 6 \mathrm{~K}$ for the near-surface maximum). This incongruent inner-core perturbation temperature structure almost certainly results from the lack of horizontal and vertical resolution in the AMSU-A data; the six channels (4-9) utilized to construct this composite perform temperature retrievals at horizontal resolution of $48 \mathrm{~km}$ at nadir and have weighting functions that are maximized at heights of $\sim 1,5,10,12,15$, and $17.5 \mathrm{~km}$, respectively. Based on this composite, it is clear that the AMSU-A temperature retrievals are inadequate for observing the inner-core temperature structure of Edouard.

\section{d. Analysis of the simulated warm core: Comparison to observations}

Although both the horizontal and tangential wind composite comparisons between the P-3 data and the WRF-EnKF ensemble were mostly favorable (Figs. 2, 3), Edouard's simulated inner-core temperature structure should also be verified before more in-depth analysis is performed. Figure 6 a shows the azimuthal-mean vertical cross section of perturbation temperature for the GOOD_LATE members at 0000 UTC 17 September, which coincides with the approximate midpoint of the 16-17 September HS3 flight. The height of the maximum perturbation temperature in the eye region in the GOOD_LATE composite is $\sim 6 \mathrm{~km}$, which agrees fairly well with the height of the lowest perturbation temperature maximum in the dropsondes. However, unlike in the innermost dropsondes, there is only one distinct maximum in perturbation temperature in the eye, the radial extent of this maximum (perturbations of at least $8 \mathrm{~K}$ ) is only $\sim 30 \mathrm{~km}$, and the vertical extent of these perturbations is confined to below $9 \mathrm{~km}$.

The overall vertical structure of composite GOOD_LATE inner-core temperature is somewhat consistent with the dropsonde data, as the region of the most significant perturbation temperatures (at least $7 \mathrm{~K}$ ) extends upward from a height of $\sim 4 \mathrm{~km}$, and the strength of the maximum in temperature perturbation is $\sim 10-12 \mathrm{~K}$ in both composites (Figs. 6a,b). To more quantitatively compare the inner-core temperature structure of GOOD_LATE and the dropsondes, perturbation temperatures averaged within a $50-\mathrm{km}$ radius and over various altitude ranges are calculated throughout the period of the HS3 flight (Figs. 7a-c). At the beginning of the HS3 flight ( 1500 UTC 16 September; $123 \mathrm{~h}$ ), the observed innercore perturbation temperatures were warmer $(\sim 4 \mathrm{~K})$ than in the GOOD_LATE composite in all three layers. However, by $6 \mathrm{~h}$ into the flight ( 2100 UTC 16 September; $129 \mathrm{~h}$ ) as Edouard began to weaken, the dropsondes and the GOOD_LATE members are in better agreement (primarily within a standard deviation of each other) and remain so for the remainder of the flight, with layeraveraged inner-core perturbation temperatures of $\sim 6-7 \mathrm{~K}$ in the low to midlevels $(4-6 \mathrm{~km})$ and $\sim 7-9 \mathrm{~K}$ in the mid- to upper levels (6-8 and 8-10 km).

A scatterplot of the height of the temperature maximum as a function of radius for dropsondes and the GOOD_LATE composite (Fig. 7d) expands upon the comparisons of the strength of the maximum inner-core perturbation temperature in the selected layers. The maximum perturbation temperature in the GOOD_LATE composite within a $10-\mathrm{km}$ radius is $\sim 10 \mathrm{~K}$ at a height of $6 \mathrm{~km}$, while the dropsondes measured a slightly stronger and higher maximum perturbation temperature ( $\sim 11-12 \mathrm{~K}$ at a height of $\sim 8 \mathrm{~km}$ ). The height of the temperature maximum increases with radius, while the strength decreases in both the dropsondes and the GOOD_LATE composite. Although the temperaturemaxima heights are in agreement between the two datasets at all radii outward of $10 \mathrm{~km}$, the GOOD_LATE perturbation temperature maxima are $\sim 2-3 \mathrm{~K}$ cooler than those measured by the dropsondes. Despite some minor discrepancies, the available observations of Edouard's inner-core temperature structure obtained throughout the 16-17 September HS3 flight compare favorably with the GOOD_LATE composite, which allows for additional analysis of the development of the warm core within the WRF-EnKF ensemble.

\section{e. Analysis of the simulated warm core: Relationship to $R I$}

The 10-member composite groups from the WRF-EnKF ensemble are now utilized to explore the relationship between Edouard's inner-core temperature structure and RI-onset time. Figure 8 shows radius-height cross sections of azimuthal-mean perturbation temperature for the GOOD_EARLY (Fig. 8a), GOOD (Fig. 8b), GOOD_LATE (Fig. 8c), and POOR (Fig. 8d) composite groups at 0000 UTC 17 September (132h), which coincides with the midpoint of the HS3 flight just after Edouard's peak intensity. GOOD_EARLY and GOOD members had respectively completed their intensification about 24 and $12 \mathrm{~h}$ before this time, and the two composites have fairly similar perturbation temperature structures. A distinct and relatively strong maximum in perturbation temperature of $\sim 10 \mathrm{~K}$ is present in the midlevels in both composites, although this maximum is slightly higher ( $\sim 7 \mathrm{~km}$ rather than $\sim 6 \mathrm{~km}$ ), and the radial extent of the $10 \mathrm{~K}$ contour is slightly larger in GOOD_EARLY ( $\sim 20 \mathrm{~km}$ rather than $\sim 10 \mathrm{~km}$ ).

As the other composites differ in evolutionary stages, more discrepancy exists in their inner-core temperature 



FIG. 7. AVAPS dropsonde (red; binned every $3 \mathrm{~h}$ ) and GOOD_LATE composite (blue) inner-core (within $50 \mathrm{~km}$ from Edouard's surface center) perturbation temperature (K) evolutions for the times in which the dropsondes were deployed (1500 UTC 16 Sep-0900 UTC 17 Sep 2014; 123-141 h) for various layer-averaged altitude ranges: (a) 4-6, (b) 6-8, and (c) 8-10 km. Azimuthal-mean temperature averaged over a 300-700-km radius from Edouard's surface center is again used as a reference profile. In (a)-(c) shaded regions show \pm 1 standard deviation from the mean. (d) Scatterplot of the height of the maximum perturbation temperature (300-700-km environmental temperature reference profile; filled markers every $0.5 \mathrm{~K}$ ) by radius for the inner-core AVAPS dropsondes (circles) and the GOOD_LATE composite (squares).

structures at this time. At $132 \mathrm{~h}$, the GOOD_LATE members have just reached their peak intensities but are $\sim 15 \mathrm{kt}$ weaker than the GOOD_EARLY or GOOD members at their peak intensities (Fig. 1c). This difference is reflected in the perturbation temperature structures; the magnitude of the warm core is $\sim 1.5 \mathrm{~K}$ cooler in GOOD_LATE, and the region of most significant perturbation temperature (at least $\sim 8 \mathrm{~K}$ ) does not extend upward as high $(\sim 8.5 \mathrm{~km}$ as opposed to $\sim 10.5 \mathrm{~km}$ ). In addition, the warming is not as deep, as perturbation temperatures exceeding $4 \mathrm{~K}$ do not extend above $12 \mathrm{~km}$ (Fig. 8c). Finally, although some of the POOR members begin to intensify toward the end of the 7-day simulation window, these late-developing members have only just begun intensification at $132 \mathrm{~h}$, and a developing warm core at $\sim 7 \mathrm{~km}$ of $\sim 4 \mathrm{~K}$ is present (Fig. 8d).
Additional insight on warm-core evolution can be derived from the availability of WRF-EnKF ensemble output across the simulation window. Figure 9 shows the 7-day evolution of the inner-core area-averaged (within a radius of $25 \mathrm{~km}$ ) perturbation temperature vertical structure for the four composite groups. For the developing composites (GOOD_EARLY, GOOD, and GOOD_LATE), the RI-onset times of the respective composites are also indicated; the POOR members do not significantly intensify in the simulation window. All composite groups initially have weak midlevel innercore perturbation temperatures $(<2 \mathrm{~K})$, as the members are only of tropical depression or weak tropical storm strength, and substantial warming has not yet occurred throughout the vortex. In the GOOD_EARLY and GOOD composites (Figs. 9a,b), some warming (average perturbation temperatures of $\sim 3 \mathrm{~K}$ ) is evident $\sim 24 \mathrm{~h}$ 

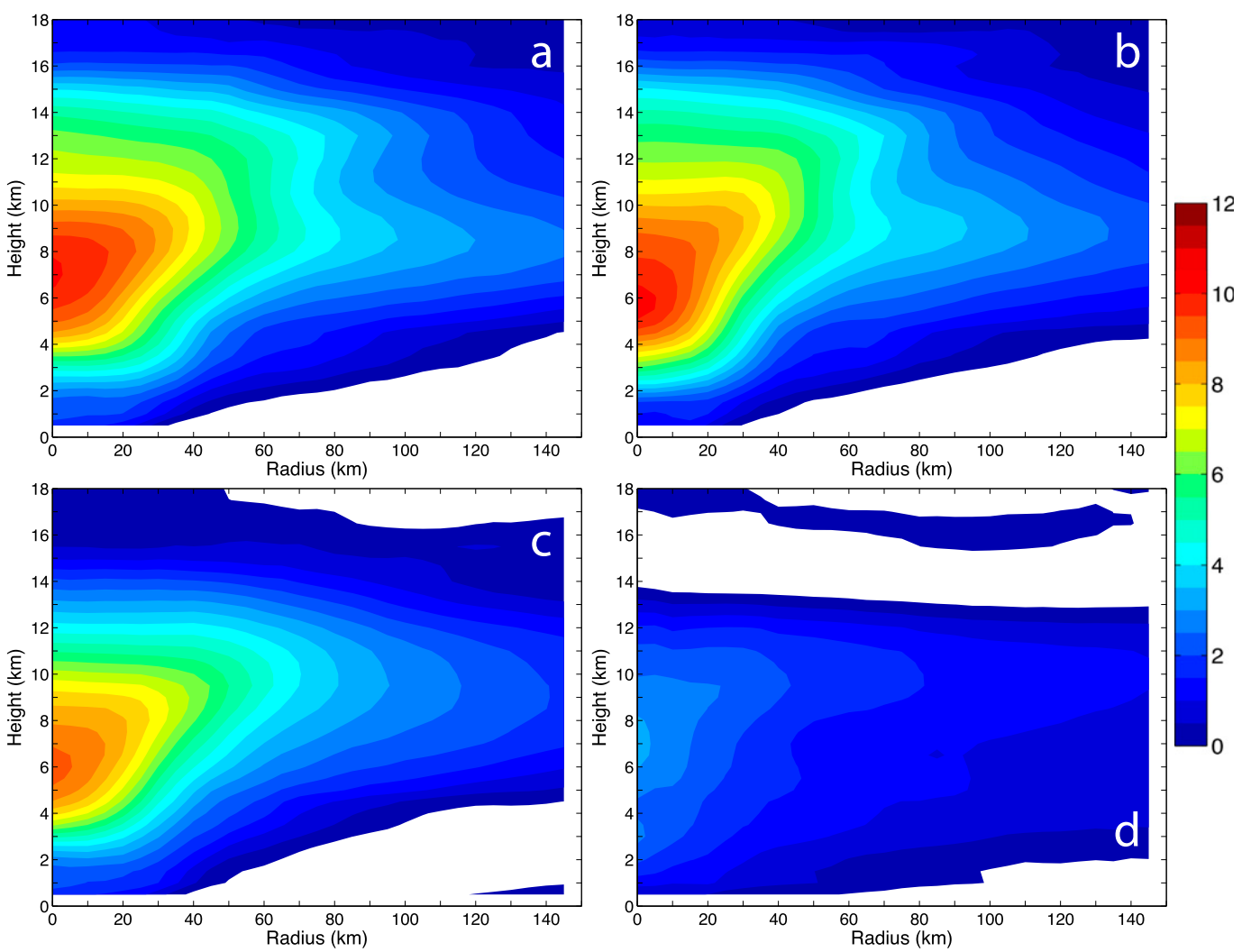

FIG. 8. As in Fig. 6, but for the (a) GOOD_EARLY, (b) GOOD, (c), GOOD_LATE, and (d) POOR composites at 0000 UTC 17 Sep 2014

prior to RI onset in the low to midlevels $(\sim 4-6 \mathrm{~km})$. In the GOOD_LATE composite (Fig. 9c), a similar pattern of warming begins up to $48 \mathrm{~h}$ prior to RI onset ( $\sim 48 \mathrm{~h}$; as in GOOD); however, onset of RI is not imminent, and the moderate warming is confined to below $6 \mathrm{~km}$ until just prior to RI ( $\sim 96 \mathrm{~h})$.

Approximately $3-6 \mathrm{~h}$ prior to RI in all three developing composites (Figs. 9a-c), a region of moderate warming (perturbation temperatures of at most $4 \mathrm{~K}$ ) extends upward through $8-10 \mathrm{~km}$. Rapid deep-layer warming occurs as the RI process begins. This signal occurs approximately in tandem with the intensification process, suggesting that this upper-level warming is not a trigger of RI; this possibility will be explored in more detail below. As intensification proceeds in the GOOD_EARLY, GOOD, and GOOD_LATE composites, warming occurs throughout most of the vortex $(\sim 2-10 \mathrm{~km})$ over the first $24 \mathrm{~h}$ of RI, with maximum perturbation temperatures of $\sim 7 \mathrm{~K}$ present in the midlevels $(6-8 \mathrm{~km})$.

By $48-72 \mathrm{~h}$ after RI onset has begun in the developing composites, the overall maximum temperature perturbation $(\sim 9-11 \mathrm{~K})$ has developed at a height of $\sim 7-8 \mathrm{~km}$. The maximum temperature perturbation in each of the composites has not only increased in magnitude over time, but the height of the maximum warming has also steadily increased from $\sim 3-5 \mathrm{~km}$ prior to RI onset upward to $\sim 7-8 \mathrm{~km}$ after intensification. Throughout this period, as Edouard is steadily intensifying, warming has become more prevalent throughout the entirety of the vertical column, with perturbation temperatures of at least $\sim 4 \mathrm{~K}$ approaching $14-16 \mathrm{~km}$ in GOOD_EARLY and GOOD. However, this upper-level warming is likely a consequence of the significant intensification that Edouard is undergoing throughout this period, as these perturbation temperatures do not develop at these heights until $24-48 \mathrm{~h}$ after RI onset.

Throughout the 7-day simulation window, significant inner-core perturbation temperatures do not develop in POOR (Fig. 9d), as significant intensification does not occur in these members. A developing warm core becomes apparent in the last $24 \mathrm{~h}$ of the simulation (144-168 h), as about half of the POOR members begin intensifying (Figs. 1b,c). However, the intensification is in its early stages, and the simulation would need to be further extended to examine the perturbation temperature structure evolution in more detail. 

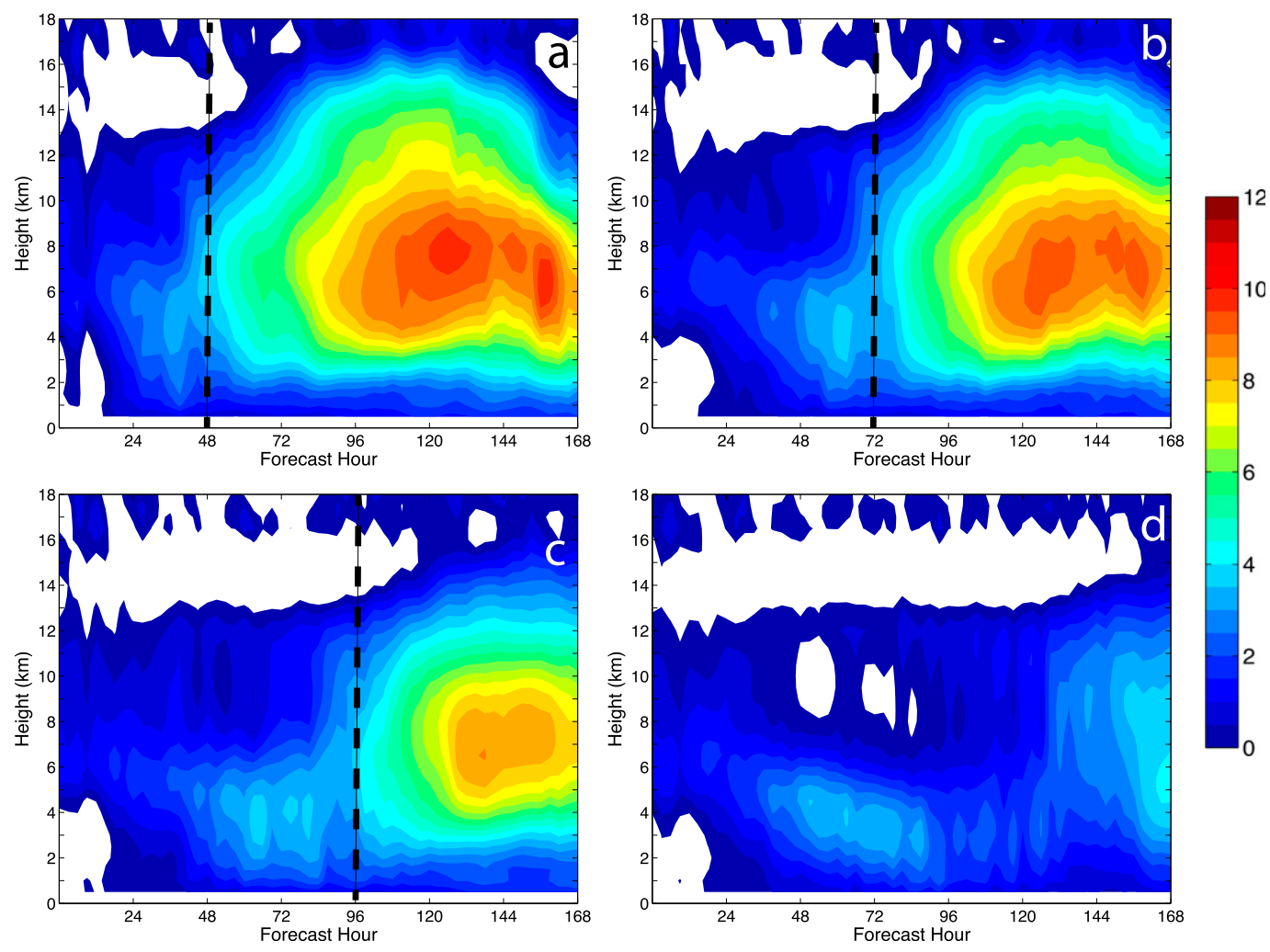

FIG. 9. Evolution of the area-averaged (within $25 \mathrm{~km}$ of the surface center) perturbation temperature vertical structure (contours filled every 0.5 K) for the (a) GOOD_EARLY, (b) GOOD, (c) GOOD_LATE, and (d) POOR composites. The dashed black line in (a)-(c) corresponds to the RI-onset time of each respective composite group; RI onset does not occur in the POOR composite.

\section{f. Analysis of the simulated warm core: Ensemble composite group variability}

The WRF-EnKF ensemble also allows for the analysis of the variability of the development of Edouard's warm core for ensemble members that have a very similar intensity evolution (e.g., within the composite groups). Figure 10 shows radius-height cross sections of azimuthal-mean temperature perturbation for nine randomly chosen members (out of 10 for ease of presentation) of the GOOD composite group just after peak intensity (0000 UTC 17 September). The innercore temperature structures of the individual GOOD members have some broad similarities to the GOOD composite temperature structure at this time (Fig. 8b). The maximum inner-core temperature perturbations are located in the midlevels (primarily $\sim 6 \mathrm{~km}$ ), while perturbations of at least $8 \mathrm{~K}$ extend $\sim 20 \mathrm{~km}$ radially outward in the vicinity of the maximum.

However, variability in the precise height and strength of the maximum temperature perturbations across the members is notably present. For example, within the temperature structures of the nine members, the height of the maximum perturbation temperature can occur as low as $5 \mathrm{~km}$ (Figs. 10b,e,h) or as high as $9 \mathrm{~km}$ (Fig. 10g), while the strength of this maximum varies from as weak as $9 \mathrm{~K}$ (Fig. $10 \mathrm{~g}$ ) to as strong as $12 \mathrm{~K}$ (Figs. 10a,b). It should also be noted that none of the members have upper-level $(>10 \mathrm{~km})$ perturbation temperature maxima, as has been seen in numerous previous modeling studies. In addition, at the height of the perturbation temperature maxima, the radial extent of the most significant warming does not vary as substantially. However, throughout the profile, perturbation temperatures of at least $7.5 \mathrm{~K}$ can at times be confined to within $25 \mathrm{~km}$ of the surface center (Fig. 10e), but they can also extend as much as $40 \mathrm{~km}$ outward (Figs. 10b,h,i).

Figure 11 shows the evolution of the area-averaged (within $25 \mathrm{~km}$ radius) perturbation temperature vertical structure for the same nine randomly chosen GOOD members whose radius-height cross sections of perturbation temperature are in Fig. 10. The storms in these members undergo slow yet steady intensification over the first $72 \mathrm{~h}$ before a period of RI begins, coincident with the best track RI onset (Figs. 1b,c). Variations in the exact timing of RI onset across the members of the GOOD composite group are limited to $6 \mathrm{~h}$ or less; 



Radius $(\mathrm{km})$

FIG. 10. As in Fig. 6, but for 9 (randomly chosen) of the 10 members of the GOOD composite group at 0000 UTC 17 Sep 2014.

therefore, the composite RI-onset time is indicated in all panels in Fig. 11. As in the comparisons between the radius-height cross sections of perturbation temperature, the evolution of both the composite (Fig. 9b) and the individual members of GOOD share some general characteristics. Little to no warming is present over the first $24 \mathrm{~h}$ throughout the vertical column, as the members do not strengthen appreciably over this period. In addition, as RI onset is approached between 24 and $72 \mathrm{~h}$, evidence of moderate warming exists in most of the ensemble members (and as a result, in the composite) in the low to midlevels $(2-6 \mathrm{~km})$, and stronger perturbation temperatures (at least $8 \mathrm{~K}$ ) do not develop until $24 \mathrm{~h}$ after RI has begun.

Despite these general similarities in inner-core perturbation temperature development, variability in the vertical temperature structure evolution is also present among the GOOD composite members. The moderate warming (mostly less than $5 \mathrm{~K}$ ) that is consistently present in the GOOD members prior to RI is primarily confined to heights below $6 \mathrm{~km}$ but can occur as high as $8 \mathrm{~km}$ (Fig. 11g). In addition, the magnitude of this pre-RI warming can be as weak as $3 \mathrm{~K}$ (Fig. 11c), or as strong as $6.5 \mathrm{~K}$ (Fig. 11i). In the $24 \mathrm{~h}$ after RI onset, nearly all of the GOOD members have perturbation temperature structures that steadily increase in magnitude up to $6-8 \mathrm{~K}$ while extending upward with height through $10 \mathrm{~km}$. Over the next $24-48 \mathrm{~h}$, the maxima in perturbation temperature develop as the members approach their peak intensities and subsequently begin to decay (Figs. 1b,c). However, differences are present in the evolution of the heights at which the maxima exist. In some of the members, perturbation temperatures of at least $9 \mathrm{~K}$ first appear $\sim 24 \mathrm{~h}$ after RI onset at heights between 4 and $6 \mathrm{~km}$ and steadily increase upward to $\sim 8 \mathrm{~km}$ within $\sim 60 \mathrm{~h}$ after RI onset (Figs. 11b,d,f). Other members see the maxima more abruptly rise to $\sim 8 \mathrm{~km}$ about $48 \mathrm{~h}$ after RI begins (Figs. 11e,g). Finally, the stronger perturbation temperatures in the majority of the rest of members develop at heights of $6-8 \mathrm{~km}$ and are maintained at this level throughout this period (Figs. 11a,c,i). 

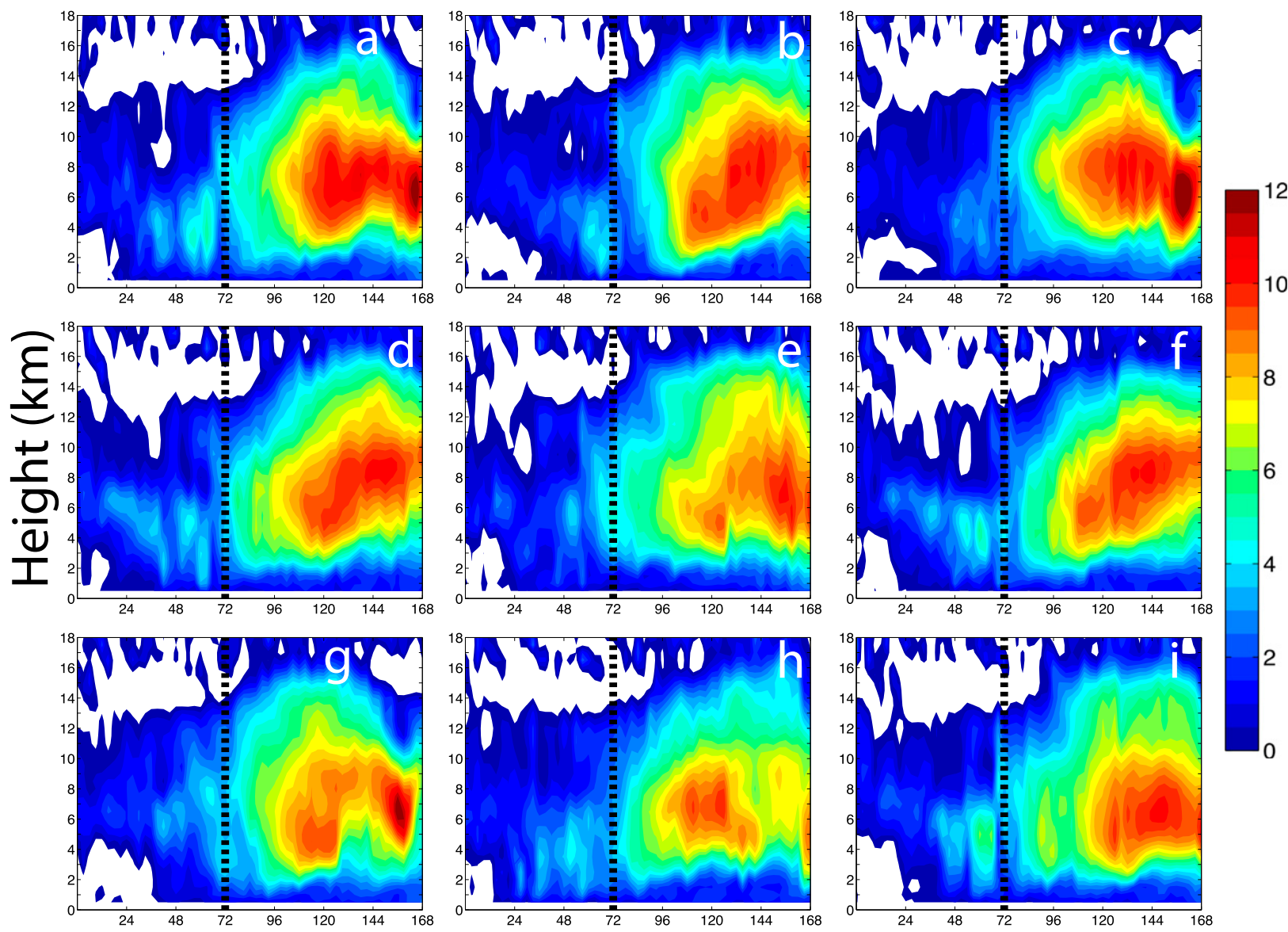

\section{Forecast Hour}

FIG. 11. As in Fig. 9, but for 9 (as in Fig. 10) of the 10 members of the GOOD composite group. Black dashed lines indicate the mean RI-onset time of GOOD.

Factors contributing to the differences in the innercore temperature structures are next briefly explored. Comprehensive potential temperature budget analyses performed in Stern and Zhang (2013a,b) showed that perturbation temperature maxima are typically confined to the midlevels because of a secondary maximum in static stability. Meanwhile, the upperlevel descent maximum is coincident with a minimum in static stability, which prevents concentrated warming at these heights. In addition, in TCs embedded in moderate vertical wind shear environments (such as Edouard), increased mixing at the eye-eyewall interface is likely. However, strong inertial stability of the vortex can allow for parcels to remain in the eye for several days, influencing the inner-core temperature structure. Following the Stern and Zhang studies, the evolutions of vertical velocity, static, and inertial stability are examined for a few GOOD members (not shown). All of these members maximize descent in the upper levels and have static stability profiles with secondary maxima in the midlevels and minima in the upper levels, which produce a midlevel warm core. However, variability is present in these evolutions as well, as larger magnitudes of midlevel static stability tend to be associated with more significant midlevel perturbation temperature maxima, while stronger and deeper vortices (as indicated by inertial stability) are typically associated with stronger warm cores. These relationships explain some of the variability present in the GOOD inner-core temperature structures, though it should be noted that these variables are only weakly correlated (not shown).

Significant variation in the inner-core perturbation temperature evolution within GOOD (with the strongest warming occurring well after RI onset) despite very similar intensity evolutions suggests that changes in the height and strength of the maximum perturbation temperature are not necessarily associated with TC intensity or subsequent intensity trends. This hypothesis will be explored quantitatively in the next section. 

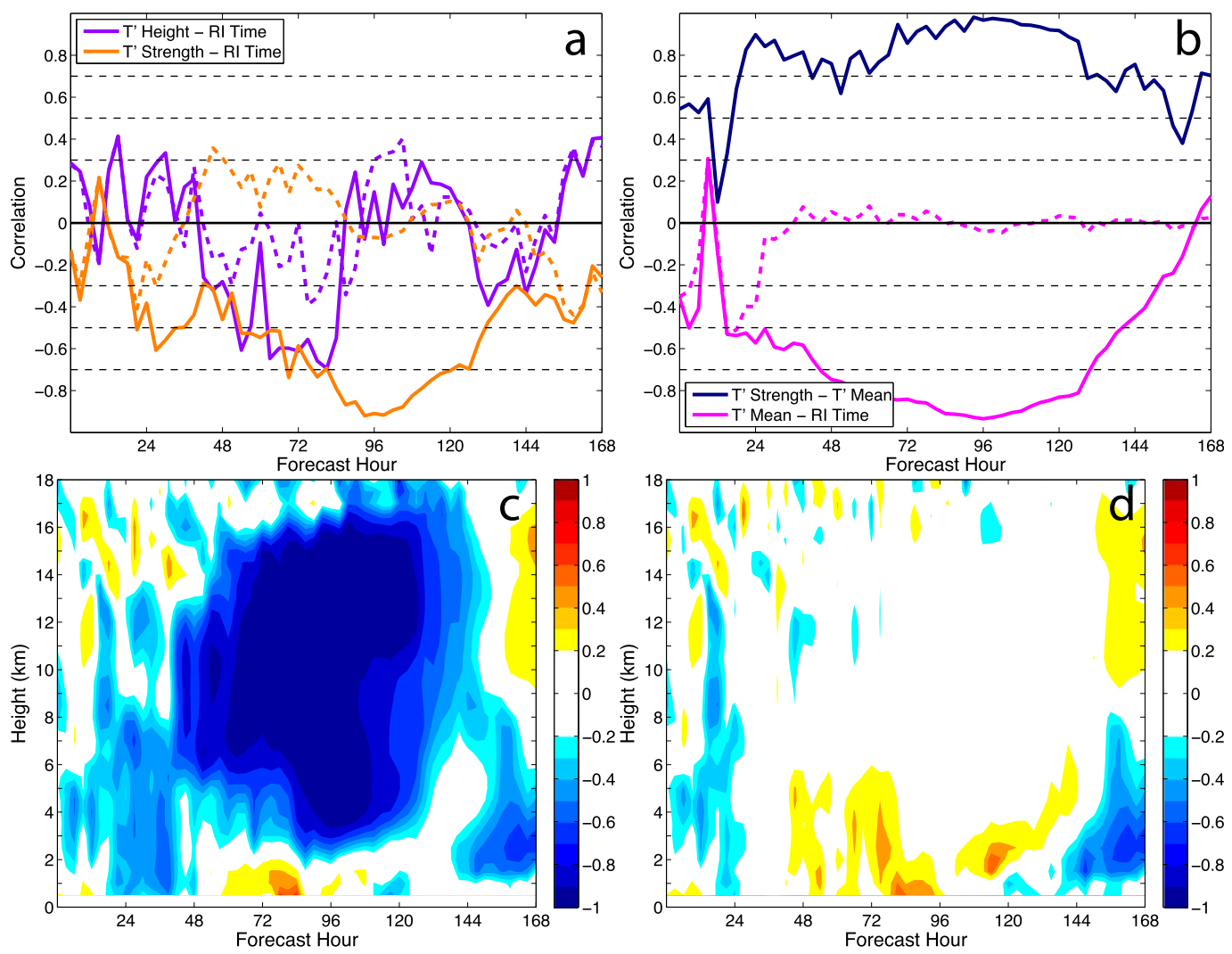

FIG. 12. (a) Evolution of the correlation (solid) and part correlation controlling for minimum SLP (dashed) between the RI times of the 30 developing composite group members and both the height (purple) and the strength of the maximum perturbation temperature (orange). (b) As in (a), but for the vertically averaged inner-core (within $25 \mathrm{~km}$ of the surface center) perturbation temperature (magenta). Correlation between the vertically averaged inner-core perturbation temperature and the strength of the maximum perturbation temperature is also plotted (dark blue). (c) Time-height correlation between the strength of the maximum perturbation temperature and the RI-onset time of the 30 members of the developing composite groups. (d) As in (c), but for the part correlation controlling for the current intensity (minimum SLP).

\section{g. Analysis of the simulated warm core: Correlation analyses}

This section uses correlation analyses to quantitatively examine the potential relationships between the perturbation temperature structure and the TC intensity. Figure 12a shows the correlation between both the height and the strength of the maxima in perturbation temperature and the RI-onset times for the 30 members of the developing composite groups. Both correlations are insignificant over the first $24 \mathrm{~h}$, as no substantial warm core development or changes in TC intensity occur during this time. Over the next $24 \mathrm{~h}$, a weak to moderate correlation between both the height $(\sim 0.3)$ and the strength $(\sim-0.3$ to -0.5 ) of the perturbation temperature maxima and RIonset time begins to develop, as the GOOD_EARLY members approach RI and warming begins to occur in the low to midlevels of only these members.

Between 48 and $96 \mathrm{~h}$, relatively strong correlations have developed $(\sim-0.6$ to -0.7$)$ between both the height and strength of the maxima in perturbation temperature and RI onset, suggesting that stronger and higher perturbation temperature maxima occur in the members whose RI onsets occur earlier in the simulation. However, much of this signal is simply a result of the divergent RI onsets rather than a driving factor in RI. Part correlations controlling for minimum SLP can account for this divergence, as the first-order part correlation between two variables while controlling for a third variable effectively treats the third as a constant (e.g., Sippel et al. 2011). Both part correlations controlling for minimum SLP fail to exceed \pm 0.3 , indicating that essentially no relationship exists between the strength or height of the maximum perturbation temperature and the subsequent RI-onset time (Fig. 12a).

To examine whether a broader relationship exists between the overall perturbation temperature structure and RI-onset time in this ensemble, the correlation between the vertically averaged inner-core (within $25 \mathrm{~km}$ of the surface center) perturbation temperature and RI- 
onset for the 30 members of the developing composite groups is also calculated (Fig. 12b). As in the correlations between the height and the strength of the perturbation temperature maxima, little to no relationship is present over the first $24 \mathrm{~h}$. However, over the next $24 \mathrm{~h}$, a moderate to strong ( $\sim-0.5$ to -0.8$)$ correlation develops as members begin to approach RI onset, which remains strong throughout most of the simulation. However, part correlations controlling for minimum SLP drop to zero by $48 \mathrm{~h}$ (Fig. 12b), indicating that essentially all of the relationship between vertically averaged inner-core perturbation temperature and RI onset results from the divergent ensemble intensities. In addition, by $24 \mathrm{~h}$, the correlation between the vertically averaged inner-core perturbation temperature and the strength of the perturbation temperature maxima is $\sim 0.9$ (Fig. 12b), demonstrating that the behavior of the perturbation temperature maxima is strongly correlated with the broader vertical structure of the inner-core temperature.

Figure $12 \mathrm{c}$ shows the evolution of the correlation between the area-averaged (within $25-\mathrm{km}$ radius) vertical profiles of perturbation temperature magnitude and RIonset time for the members of the developing composites. Between 24 and $48 \mathrm{~h}$, a region of weak to moderate negative correlation (as much as $\sim-0.6$ ) develops between 2 and $8 \mathrm{~km}$, which is representative of the moderate warming present in the low to midlevels of the composites in the times leading up to RI (Figs. 9, 11). Over the next few days of the simulation, as the various composite groups approach their respective RI-onset times, the correlation grows very significantly throughout much of the vertical profile. This result indicates that the perturbation temperatures increase in magnitude according to earlier RI-onset times. However, when the part correlation controlling for current minimum SLP is calculated (Fig. 12d), the entirety of the significant region of correlation discussed above vanishes, reinforcing the conclusion that the relationship between the inner-core perturbation temperature structure and RI onset results from the diverging intensities in the ensemble. It is therefore unlikely that the evolution of the inner-core temperature structure could be used as a predictor of RI onset in this ensemble. Cross correlations between RI-onset time and warm core development confirm this hypothesis, as the majority of the significantly intensifying members have correlations that peak at lags of $0-6 \mathrm{~h}$ after RI onset (not shown).

\section{Summary and conclusions}

This study examines the evolution of the inner-core temperature structure of Hurricane Edouard (2014), primarily through high-altitude dropsondes deployed during the 2014 campaign of HS3 and a 60-member
WRF-EnKF simulation. This ensemble was originally a 5-day real-time forecast generated by the PSU Atlantic hurricane forecast and analysis system (extended to 7 days in this study), and the resulting ensemble wind field structures have been verified against Doppler wind analyses obtained by the NOAA P-3 aircraft and HS3 dropsondes. Composite groups based on differences in RI-onset timing [first defined in Munsell et al. (2017)] are utilized to examine the variability associated with Edouard's warm core development.

Throughout the 16-17 September HS3 flight, two distinct perturbation temperature structures were measured. The profiles of the innermost dropsondes primarily yielded multiple perturbation temperature maxima of $\sim 10-12 \mathrm{~K}$, centered at 4-6 and 7-9 km; some dropsondes have an additional maximum at $\sim 10 \mathrm{~km}$. Meanwhile, the dropsondes farther away from the surface center observed a single perturbation temperature maximum of $\sim 6-8 \mathrm{~K}$ at heights of $\sim 7-9 \mathrm{~km}$. The inner-core perturbation temperature composites of the members of GOOD_LATE, whose intensities agree with the best track during the 16-17 September flight, also compare favorably with the HS3 observations. The height of the maximum perturbation temperature at Edouard's peak intensity is slightly lower in GOOD_LATE $(\sim 6 \mathrm{~km})$ than observed, and no evidence of multiple perturbation temperature maxima is present in the innermost region of Edouard's eye. However, the overall inner-core temperature structure and the magnitude of the perturbation temperature maxima are comparable between the model composite and the observations.

Given this agreement, the increased temporal frequency of the ensemble output allows for additional insight into the development of Edouard's warm core throughout the intensification period to be obtained. Despite as much as 48-60 h of simulation time between RI onset in the GOOD_EARLY, GOOD, and GOOD_LATE members, the evolutions of Edouard's inner-core perturbation temperature have many similarities when compared in an RI-onset time-relative framework. All developing composites indicate some moderate warming $(\sim 4 \mathrm{~K})$ in the low to midlevels $(\sim 2-6 \mathrm{~km}) \sim 24-48 \mathrm{~h}$ prior to RI, but the most significant warming $(>7 \mathrm{~K})$ is present higher in the inner core $(\sim 8 \mathrm{~km})$ and does not occur until at least $24 \mathrm{~h}$ after RI begins.

Despite broad similarities in the evolution of the inner-core temperature structure of the developing composites with respect to RI-onset time, variability is present within the composite groups. The strength of the maximum inner-core perturbation temperature in the GOOD members at peak intensity varies by as much as $3 \mathrm{~K}$ (magnitudes of $\sim 9-12 \mathrm{~K}$ ), and the height at which this maximum occurs can be as low as $5 \mathrm{~km}$ or as high as $9 \mathrm{~km}$. In addition, although moderate low- to midlevel 
warming is present in nearly all of the members $\sim 24 \mathrm{~h}$ prior to RI (as in the composite), the magnitude of this warming varies by $\sim 3 \mathrm{~K}$. Approximately $24 \mathrm{~h}$ after RI has begun, as stronger inner-core warming begins to occur, the evolution of the height at which the maximum perturbation temperature occurs differs across the members of GOOD. In particular, the warm core steadily builds upward in height in some members, while other members have perturbation temperature maxima at relatively constant heights. It should be noted that unlike in the Hurricane Wilma (2005) simulation examined in Chen et al. (2011) and Chen and Zhang (2013), no evidence of an upper-tropospheric warm core is present in any of the members prior to RI, and warming at any level never serves as a trigger for RI because the most significant warming always occurs after RI onset.

Although midlevel perturbation temperature maxima always develop in the GOOD members $\sim 24 \mathrm{~h}$ after RI onset [primarily due to secondary maxima in static stability at these levels as thoroughly demonstrated in Stern and Zhang (2013b)], the causes of the variability in the warm core vertical structure within the composite group (whose members have very similar intensities) need to be explored further. There is some evidence that variations in the strength of the inner-core updrafts, the magnitude of midlevel static stability, and the strength and depth of the intensifying vortex (as measured by inertial stability) can impact the height and strength at which the maximum warming occurs, although these variables are only weakly correlated.

To further examine the relationships between innercore temperature structure and TC intensity more quantitatively, additional correlation analyses are performed. At times throughout the simulation window, the correlation between both the strength and height of the perturbation temperature maxima and RI onset approach moderate to strong values. This is mostly a result of ensemble divergence and not a causal factor for RI in the ensemble, as illustrated by insignificant part correlations controlling for current minimum SLP. These results imply that there is little to no relationship between the strength or height of the maximum perturbation temperature and subsequent TC intensity changes, consistent with Stern and Zhang (2016) and Komaromi and Doyle (2017). In addition, the correlation between RI onset and the moderate warming in the low to midlevels that is observed $\sim 24 \mathrm{~h}$ prior to RI also becomes insignificant when controlling for current intensity. This similarly suggests that thermodynamic changes in the inner core of Edouard likely occur either in tandem with or after intensification has already commenced and are therefore not a useful predictor of RI onset in this ensemble.
The conclusion in this study that inner-core temperature structure is unrelated to future intensity changes in the Edouard ensemble is similar to conclusions reached by Stern and Zhang (2016) and Komaromi and Doyle (2017), which used dropsondes and a deterministic simulation from a single TC (Hurricane Earl 2010) and high-altitude dropsondes from a variety of TCs sampled during HS3 to demonstrate this same point. In addition, despite very similar intensity evolutions within the GOOD composite group, considerable variability exists in the exact temperature structure of the inner core, as significant differences are present in the precise height and strength of the perturbation temperature maxima. Therefore, the intensity of the TC does not dictate the exact details of the vertical profile of inner-core temperature structure.

Acknowledgments. This work is supported by the NASA New Investigator Program (Grants NNX12AJ79G, NNX15AF38G, and NNX16AI21G), the Office of Naval Research (Grant N000140910526), the National Science Foundation (Grant AGS-1305798), NASA's Hurricane Science Research Program (HSRP), the Hurricane and Severe Storm Sentinel (HS3) investigation under NASA's Earth Venture Program, and NOAA's Hurricane Forecast Improvement Program (HFIP). The research was performed in part while the first author was appointed as a NASA Postdoctoral Program fellow at the Goddard Space Flight Center (GSFC), administered by USRA through a contract with NASA. Computing was performed at the Texas Advanced Computing Center (TACC). We thank Yonghui Weng for conducting the PSU WRF-EnKF analysis and forecasting of the event, Derrick Herndon for providing the University of Wisconsin/CIMSS processed AMSU-A satellite data, and Daniel Stern and two anonymous reviewers for beneficial comments on an earlier version of the manuscript. We also thank NOAA/HRD for making the P-3 Doppler radar analyses available and Daniel Stern for providing code to help visualize this data.

\section{REFERENCES}

Barker, D. M., W. Huang, Y.-R. Guo, A. J. Bourgeois, and Q. N. Xiao, 2004: A three-dimensional variational data assimilation system for MM5: Implementation and initial results. Mon. Wea. Rev., 132, 897-914, https://doi.org/10.1175/1520-0493(2004)132<0897: ATVDAS $>2.0 . \mathrm{CO} ; 2$.

Braun, S. A., and Coauthors, 2013: NASA's Genesis and Rapid Intensification Processes (GRIP) field experiment. Bull. Amer. Meteor. Soc., 94, 345-363, https://doi.org/10.1175/ BAMS-D-11-00232.1.

_ , P. A. Newman, and G. M. Heymsfield, 2016: NASA's Hurricane and Severe Storm Sentinel (HS3) investigation. Bull. Amer. Meteor. Soc., 97, 2085-2102, https://doi.org/10.1175/ BAMS-D-15-00186.1. 
Chen, H., and D.-L. Zhang, 2013: On the rapid intensification of Hurricane Wilma (2005). Part II: Convective bursts and the upper-level warm core. J. Atmos. Sci., 70, 146-162, https://doi. org/10.1175/JAS-D-12-062.1.

— rapid intensification of Hurricane Earl (2010) using the HWRF system. J. Atmos. Sci., 72, 531-550, https://doi.org/ 10.1175/JAS-D-14-0097.1.

—, D.-L. Zhang, J. Carton, and R. Atlas, 2011: On the rapid intensification of Hurricane Wilma (2005). Part I: Model prediction and structural changes. Wea. Forecasting, 26, 885901, https://doi.org/10.1175/WAF-D-11-00001.1.

Dunion, J. P., 2011: Rewriting the climatology of the tropical North Atlantic and Caribbean Sea atmosphere. J. Climate, 24, 893908, https://doi.org/10.1175/2010JCLI3496.1.

Durden, S. L., 2013: Observed tropical cyclone eye thermal anomaly profiles extending above $300 \mathrm{hPa}$. Mon. Wea. Rev., 141, 4256-4268, https://doi.org/10.1175/MWR-D-13-00021.1.

Fang, J., O. Pauluis, and F. Zhang, 2017: Isentropic analysis on the intensification of Hurricane Edouard (2014). J. Atmos. Sci., 74, 4177-4197, https://doi.org/10.1175/JAS-D-17-0092.1.

Gamache, J. F., J. S. Griffin Jr., P. P. Dodge, and N. F. Griffin, 2004: Automatic Doppler analysis of three-dimensional wind fields in hurricane eyewalls. 26th Conf. on Hurricanes and Tropical Meteorology, Miami, FL, Amer. Meteor. Soc., 5D.4, http:// ams.confex.com/ams/pdfpapers/75806.pdf.

Halverson, J. B., J. Simpson, G. Heymsfield, H. Pierce, T. Hock, and L. Ritchie, 2006: Warm core structure of Hurricane Erin diagnosed from high altitude dropsondes during CAMEX-4. J. Atmos. Sci., 63, 309-324, https://doi.org/10.1175/JAS3596.1.

Hawkins, H. F., and D. T. Rubsam, 1968: Hurricane Hilda, 1964. II: Structure and budgets of the hurricane on October 1, 1964. Mon. Wea. Rev., 96, 617-636, https://doi.org/10.1175/ 1520-0493(1968)096<0617:HH>2.0.CO;2.

— , and S. M. Imbembo, 1976: The structure of a small, intense hurricane-Inez 1966. Mon. Wea. Rev., 104, 418-442, https://doi.org/ 10.1175/1520-0493(1976)104<0418:TSOASI>2.0.CO;2.

Hock, T., K. Young, and H. Voemel, 2017: Hurricane and Severe Storm Sentinel (HS3) Global Hawk Advanced Vertical Atmospheric Profiling System (AVAPS) Dropsonde System V2. NASA Global Hydrology Center DAAC, Hunstville, AL, accessed 16 September 2016, https://doi.org/10.5067/HS3/ AVAPS/DROPSONDE/DATA202.

Jordan, C. L., 1958: Mean soundings for the West Indies area. J. Meteor., 15, 91-97, https://doi.org/10.1175/1520-0469(1958)015<0091: MSFTWI $>2.0 . \mathrm{CO} ; 2$.

Knaff, J. A., S. A. Seseske, M. DeMaria, and J. L. Demuth, 2004: On the influences of vertical wind shear on symmetric tropical cyclone structure derived from AMSU. Mon. Wea. Rev., 132, 2503-2510, https://doi.org/10.1175/1520-0493(2004)132<2503: OTIOVW $>2.0 . \mathrm{CO} ; 2$.

Komaromi, W. A., and J. D. Doyle, 2017: Tropical cyclone outflow and warm core structure as revealed by HS3 dropsonde data. Mon. Wea. Rev., 145, 1339-1359, https://doi.org/10.1175/ MWR-D-16-0172.1.

La Seur, N. E., and H. F. Hawkins, 1963: An analysis of Hurricane Cleo (1958) based on data from research reconnaissance aircraft. Mon. Wea. Rev., 91, 694-709, https://doi.org/10.1175/ 1520-0493(1963)091<0694:AAOHCB > 2.3.CO;2.

Melhauser, C., F. Zhang, Y. Weng, Y. Jin, H. Jin, and Q. Zhao, 2017: A multiple-model convection-permitting ensemble examination of the probabilistic prediction of tropical cyclones: Hurri- canes Sandy (2012) and Edouard (2014). Wea. Forecasting, 32, 665-688, https://doi.org/10.1175/WAF-D-16-0082.1.

Munsell, E. B., and F. Zhang, 2014: Prediction and uncertainty of Hurricane Sandy (2012) explored through a real-time cloud-permitting ensemble analysis and forecast system assimilating airborne Doppler radar observations. J. Adv. Model. Earth Syst., 6, 38-58, https://doi.org/10.1002/ 2013 MS000297.

,-- - and D. P. Stern, 2013: Predictability and dynamics of a nonintensifying tropical storm: Erika (2009). J. Atmos. Sci., 70, 2505-2524, https://doi.org/10.1175/JAS-D-12-0243.1.

_ J. A. Sippel, S. A. Braun, Y. Weng, and F. Zhang, 2015: Dynamics and predictability of Hurricane Nadine (2012) evaluated through convection-permitting ensemble analysis and forecasts. Mon. Wea. Rev., 143, 4514-4532, https://oi.org/ 10.1175/MWR-D-14-00358.1.

— F. Zhang, J. A. Sippel, S. A. Braun, and Y. Weng, 2017: Dynamics and predictability of the intensification of Hurricane Edouard (2014). J. Atmos. Sci., 74, 573-595, https://doi. org/10.1175/JAS-D-16-0018.1.

Ohno, T., and M. Satoh, 2015: On the warm core of a tropical cyclone formed near the tropopause. J. Atmos. Sci., 72, 551-571, https://doi.org/10.1175/JAS-D-14-0078.1.

Revercomb, H. E., and J. K. Taylor, 2017: Hurricane and Severe Storm Sentinel (HS3) Scanning High-Resolution Interferometer Sounder (S-HIS). NASA Global Hydrology Center DAAC, Huntsville, AL, accessed 4 April 2016, https:// doi.org/10.5067/HS3/SHIS/DATA201.

Rios-Berrios, R., R. D. Torn, and C. A. Davis, 2016: An ensemble approach to investigate tropical cyclone intensification in sheared environments. Part I: Katia (2011). J. Atmos. Sci., 73, 71-93, https://doi.org/10.1175/JAS-D-15-0052.1.

Rogers, R. F., and Coauthors, 2013: NOAA's Hurricane Intensity Forecasting Experiment: A progress report. Bull. Amer. Meteor. Soc., 94, 859-882, https://doi.org/10.1175/BAMS-D-12-00089.1.

_ J. A. Zhang, J. Zawislak, H. Jiang, G. R. Alvey, E. J. Zipser, and S. N. Stevenson, 2016: Observations of the structure and evolution of Hurricane Edouard (2014) during intensity change. Part II: Kinematic structure and the distribution of deep convection. Mon. Wea. Rev., 144, 3355-3376, https:// doi.org/10.1175/MWR-D-16-0017.1.

Sippel, J. A., S. A. Braun, and C.-L. Shie, 2011: Environmental influences on the strength of Tropical Storm Debby (2006). J. Atmos. Sci., 68, 2557-2581, https://doi.org/10.1175/2011JAS3648.1.

Skamarock, W. C., and Coauthors, 2008: A description of the Advanced Research WRF version 3. NCAR Tech. Note NCAR/TN-475+STR, 113 pp., https://doi.org/10.5065/ D68S4MVH.

Stern, D. P., and D. S. Nolan, 2009: Reexamining the vertical structure of tangential winds in tropical cyclones: Observations and theory. J. Atmos. Sci., 66, 3579-3600, https://doi.org/ 10.1175/2009JAS2916.1.

— , and — 2012: On the height of the warm core in tropical cyclones. J. Atmos. Sci., 69, 1657-1680, https://doi.org/10.1175/ JAS-D-11-010.1.

— , and F. Zhang, 2013a: How does the eye warm? Part I: A potential temperature budget analysis of an idealized tropical cyclone. J. Atmos. Sci., 70, 73-90, https://doi.org/10.1175/ JAS-D-11-0329.1.

_ and _ 2013b: How does the eye warm? Part II: Sensitivity to vertical wind shear and a trajectory analysis. J. Atmos. Sci., 70, 1849-1873, https://doi.org/10.1175/JAS-D-12-0258.1. 
, and 2016: The warm-core structure of Hurricane Earl (2010). J. Atmos. Sci., 73, 3305-3328, https://doi.org/10.1175/ JAS-D-15-0328.1.

Stewart, S. R., 2014: Tropical cyclone report: Hurricane Edouard. NOAA/National Hurricane Center Tech. Rep. AL062014, 19 pp., http://www.nhc.noaa.gov/data/tcr/ AL062014_Edouard.pdf.

Tang, X., and F. Zhang, 2016: Impacts of the diurnal radiation cycle on the formation, intensity, and structure of Hurricane Edouard (2014). J. Atmos. Sci., 73, 2871-2892, https://doi.org/ 10.1175/JAS-D-15-0283.1.

—, Z. Tan, J. Fang, Y. Q. Sun, and F. Zhang, 2017: Impacts of the diurnal radiation cycle on secondary eyewall formation. J. Atmos. Sci., 74, 3079-3098,https://doi.org/ 10.1175/JAS-D-17-0020.1.

Wang, H., and Y. Wang, 2014: A numerical study of Typhoon Megi (2010). Part I: Rapid intensification. Mon. Wea. Rev., 142, 29-48, https://doi.org/10.1175/MWR-D-13-00070.1.

Weng, Y., and F. Zhang, 2012: Assimilating airborne Doppler radar observations with an ensemble Kalman filter for convection-permitting hurricane initialization and prediction: Katrina (2005). Mon. Wea. Rev., 140, 841-859, https://doi.org/ 10.1175/2011MWR3602.1.

, and _- 2016: Advances in convection-permitting tropical cyclone analysis and prediction through EnKF assimilation of reconnaissance aircraft observations. J. Meteor. Soc. Japan, 94, 345-358, https://doi.org/10.2151/jmsj.2016-018.

Willoughby, H. E., 1990: Gradient balance in tropical cyclones. J. Atmos. Sci., 47, 265-274, https://doi.org/10.1175/1520-0469(1990)047<0265: GBITC $>2.0 . \mathrm{CO} ; 2$.

Zawislak, J., H. Jiang, G. R. Alvey, E. J. Zipser, R. F. Rogers, J. A. Zhang, and S. N. Stevenson, 2016: Observations of the structure and evolution of Hurricane Edouard (2014) during intensity change. Part I: Relationship between the thermodynamic structure and precipitation. Mon. Wea. Rev., 144, 3333-3354, https://doi.org/10.1175/MWR-D-16-0018.1.

Zhang, F., and Y. Weng, 2015: Predicting hurricane intensity and associated hazards: A five-year real-time forecast experiment with assimilation of airborne Doppler radar observations. Bull. Amer. Meteor. Soc., 96, 25-33, https://doi.org/10.1175/BAMS-D-13-00231.1.

, J. A. Sippel, Z. Meng, and C. H. Bishop, 2009: Cloudresolving hurricane initialization and prediction through assimilation of Doppler radar observations with an ensemble Kalman filter. Mon. Wea. Rev., 137, 2105-2125, https://doi.org/ 10.1175/2009MWR2645.1.

, _ _ J. F. Gamache, and F. D. Marks, 2011: Performance of convection-permitting hurricane initialization and prediction during 2008-2010 with ensemble data assimilation of inner-core airborne Doppler radar observations. Geophys. Res. Lett., 38, L15810, https://doi.org/10.1029/2011GL048469. 